\title{
Mappings of stochastic field lines in poloidal divertor tokamaks
}

\author{
S.S. Abdullaev, K.H. Finken, M. Jakubowski and M. Lehnen \\ Institut für Plasmaphysik, Forschungszentrum Jülich GmbH, EURATOM Association, \\ Trilateral Euregio Cluster, D-52425 Jülich, Germany
}

Received 5 August 2005, accepted for publication 10 November 2005

Published 15 March 2006

Online at stacks.iop.org/NF/46/S113

\begin{abstract}
Two mapping methods to study magnetic field lines near the separatrix of poloidal divertor tokamaks in the presence of external non-axisymmetric magnetic perturbations are proposed. The first mapping method is based on the Hamiltonian formulation of field line equations in the Boozer coordinates and solving it by the canonical transformation of variables (Abdullaev et al 1999 Phys. Plasmas 6 153). The second mapping is a canonical mapping near the separatrix which is constructed using the recently developed method (Abdullaev 2004 Phys. Rev. E 70 064202, Abdullaev 2005 Phys. Rev. E 72 064202). We construct the corresponding mappings for magnetic field lines in divertor tokamaks in the presence of non-axisymmetric magnetic perturbations. The mappings are applied to study the properties of open stochastic field lines near the separatrix for the wire model of the plasma. Poincaré sections, the so-called laminar and magnetic footprint plot (a contour plot of wall to wall connections lengths) in the plasma region and on the divertor plates are obtained. The quasilinear diffusion coefficients of field lines are also estimated.
\end{abstract}

PACS numbers: 52.55.Dy, 52.55.Fa, 05.45.Ac, 45.20.Jj

(Some figures in this article are in colour only in the electronic version)

\section{Introduction}

Poloidal divertor tokamaks are an important concept of the magnetic confinement of plasma (see [1]). The magnetic configuration of these tokamaks contains a magnetic surface (a magnetic separatrix) sharply separating closed field lines on nested magnetic surfaces from open field lines hitting the walls of fusion device. It has one (or two) singular points, X-points, on the poloidal section where the poloidal components of the magnetic field are zeros. A schematic view of the so-called single-null poloidal divertor is shown in figure 1. Such configurations of the magnetic field are created by one or two external current coils parallel to the plasma current, respectively. Magnetic fusion devices with a poloidal divertor provide an improved energy confinement of the plasma and divert particles and heat efficiently into divertor plates in a special volume, from where they are pumped away. The future international thermonuclear experimental reactor (ITER) is designed as a poloidal divertor tokamak.

Magnetic field lines in such a magnetic configuration are described by the Hamiltonian system with hyperbolic fixed points. The magnetic separatrix and the X-points correspond to the separatrices and the hyperbolic saddle points, respectively. Typically any small non-axisymmetric magnetic perturbations

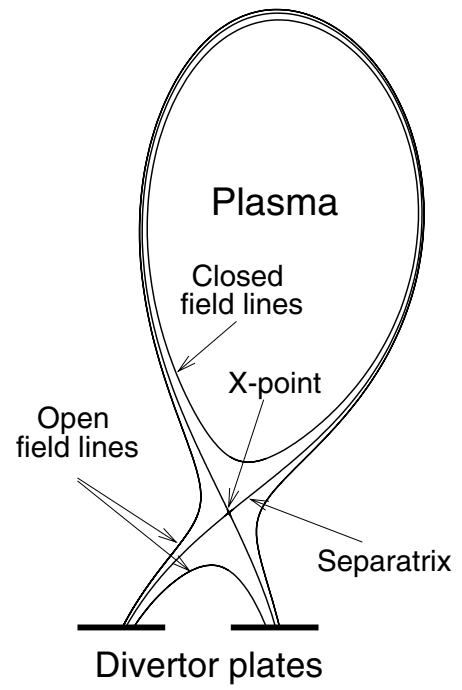

Figure 1. Schematic view of a magnetic configuration in a poloidal single-null divertor tokamak.

destroy the magnetic separatrix replacing it by the stochastic layer of field lines.

The nature of these magnetic perturbations may range from magnetic fluctuations produced by plasma instabilities, 
field errors, etc. In present day tokamaks one uses specially created external magnetic fields to null the magnetic perturbation caused by magneto hydrodynamic instabilities. Typically, magnitudes of magnetic perturbation fields are small. For instance, the amplitudes of the error field are $\delta B / B_{0} \approx 10^{-4}$ (see, e.g. [2]). Determinations of the structure of the stochastic layer and the pattern of magnetic footprints on the divertor plates are important issues in the understanding of particles and energy deposition in tokamak fusion devices [2-5]. The study of the stochastic layer near the separatrix also becomes important in the light of recent experiments in the DIII-D tokamak on the suppression of large edge-localized modes in high confinement (H-mode) plasmas affected by applied edge resonant magnetic perturbation [6].

In the early theoretical works in $[3,7,8]$ the effect of magnetic perturbations on divertor separatrix has been studied by employing the Chirikov overlapping criteria to estimate the width of the stochastic layer in the absence of divertor plates. Direct numerical integration of the equations of field lines in the DIII-D tokamak in the presence of field irregularities has been performed in [9]. The effect of magnetic perturbations created by special coils in this tokamak has been analysed in [5] (see also references therein) using a numerical code which takes into account a real magnetic geometry of the system. 'Wire' models have been used in [2,4] to study the effect of magnetic field errors on the formation of the stochastic layer near the magnetic separatrix and magnetic footprints on the divertor plates.

Mapping approaches to study magnetic field lines near the separatrix in divertor tokamaks have been developed in [10-19]. The mappings are computationally efficient and they run much faster than the numerical integration of field line equations. In [10-15] the simple mapping models, called tokamak divertor maps, are proposed to describe the field lines in poloidal divertor tokamaks. These areapreserving maps are simple algebraic difference equations. They allow us to study generic features of the structure of field lines near X-points and the magnetic footprints affected by asymmetric magnetic perturbations. However, since these simple maps are not deduced from the field line equations it is not clear how parameters of maps are related to the magnetic field configuration. It makes it difficult to apply these maps to analyze the magnetic structure of real poloidal divertor tokamaks.

A separatrix mapping approach to estimate the width of the stochastic layer formed near the magnetic separatrix has been considered in [16]. The author following [20] derived the separatrix map for field lines near the magnetic separatrix in a single null poloidal divertor tokamak. This separatrix map allowed us to plot the structure of the stochastic layer and estimate its width. In [17-19] the separatrix mapping method has been generalized to describe the field line near the X-points and on the divertor plates. The method of construction of the separatrix mapping from the equations of field lines in poloidal divertor tokamaks with arbitrary magnetic configuration has been proposed. It allowed us to obtain not only the structure of the stochastic layer but also the magnetic footprint patterns on the divertor plates.

Below we present two mapping methods to study stochastic field lines in poloidal divertor tokamaks in

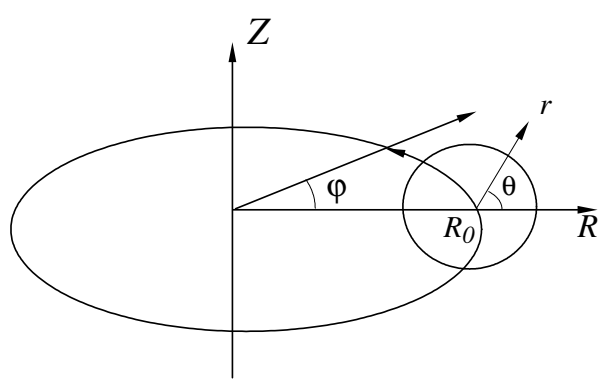

Figure 2. Geometry of a toroidal system.

the presence of non-axisymmetric magnetic perturbations. The method is based on the Hamiltonian formulation of field line equations in the Boozer coordinates and their mapping solutions obtained by canonical transformation of variables [25-27]. This method has been previously successfully applied to study the magnetic structure in the TEXTOR-DED [28] in a number of theoretical and experimental works [29-31,39]. Other computationally effective mappings are canonical mappings near the separatrix. The method of construction of these mappings is given in $[21,22]$. In this work we apply this method to study field lines near the magnetic separatrix.

The content of the work is as follows. In section 2 we recall the Hamiltonian formulation of field line equations and the generic properties of field lines near the separatrix in the absence and the presence of magnetic perturbations. Two mapping approaches to study field lines are presented in section 3 . The wire model of the plasma and the application of mapping methods to this model are described in section 4 . We study, particularly the structure of the stochastic layer of field lines near the separatrix by plotting Poincaré sections of field lines, laminar plots and magnetic footprints.

\section{Field line equations near the separatrix}

Consider a cylindrical coordinate system shown in figure 2 . The equations of magnetic field lines in this system are

$$
\frac{1}{R} \frac{\mathrm{d} Z}{\mathrm{~d} \varphi}=\frac{B_{Z}}{B_{\varphi}}, \quad \frac{1}{R} \frac{\mathrm{d} R}{\mathrm{~d} \varphi}=\frac{B_{R}}{B_{\varphi}} .
$$

The magnetic field $\boldsymbol{B}$ can be presented through the vector potential $\boldsymbol{A}(R, Z, \varphi)=\left(A_{R}, A_{\varphi}, A_{Z}\right): \boldsymbol{B}=\nabla \times \boldsymbol{A}$. Because of the gauge invariance of the vector potential one can always choose $A_{R}=0$. Then one can express the magnetic field through the components of the vector potential

$B_{R}=\frac{1}{R} \frac{\partial A_{Z}}{\partial \varphi}-\frac{\partial A_{\varphi}}{\partial Z}, \quad B_{\varphi}=-\frac{\partial A_{Z}}{\partial R}, \quad B_{Z}=\frac{1}{R} \frac{\partial R A_{\varphi}}{\partial R}$.

The $A_{Z}$ component of the vector potential determines the main toroidal component of the magnetic field $B_{\varphi}$ which typically decays inverse proportional to the radial coordinate $R: B_{\varphi} \propto R^{-1}$. In typical plasmas the deviation of the toroidal field $B_{\varphi}$ from this law due to, for instance, a diamagnetic current, is small. For this reason one can neglect a dependence of $A_{Z}$ on the $Z$ coordinate and the toroidal angle $\varphi$. We introduce canonical variables $\left(z, p_{z}\right)$ of Hamiltonian system 
related to the geometrical coordinate $(R, Z)$ and the magnetic field, $\boldsymbol{B}$, according to

$z=\frac{Z}{R_{0}}, \quad p_{z}=\frac{1}{B_{0} R_{0}} \int_{R_{0}}^{R} B_{\varphi} \mathrm{d} R=-\frac{A_{z}(R)-A_{z}\left(R_{0}\right)}{B_{0} R_{0}}$.

Then the equations for field lines (1) can be transformed to the Hamiltonian form

$$
\frac{\mathrm{d} z}{\mathrm{~d} \varphi}=\frac{\partial H}{\partial p_{z}}, \quad \frac{\mathrm{d} p_{z}}{\mathrm{~d} \varphi}=-\frac{\partial H}{\partial z} .
$$

The variables $\left(z, p_{z}\right)$ are canonical coordinate and momentum, the toroidal angle $\varphi$ plays the role of a time-like independent variable and the Hamiltonian function $H$ is determined by the normalized $\varphi$-component of the vector potential

$$
H \equiv H\left(z, p_{z}, \varphi\right)=-\frac{R A_{\varphi}}{B_{0} R_{0}^{2}} .
$$

\subsection{Field lines in equilibrium plasmas}

Consider the unperturbed case when the magnetic field is homogeneous along the toroidal angle $\varphi: A_{\varphi}=$ $A_{\varphi}(R, Z)$. The magnetic surfaces then are determined by a contour line of $R A_{\varphi}(R, Z)=$ const. The field line equations are determined by the unperturbed Hamiltonian $H_{0}\left(z, p_{z}\right)=-R\left(p_{z}\right) A_{\varphi}\left(R\left(p_{z}\right), R_{0} z\right) / R_{0}^{2} B_{0}$. The toroidal flux $\psi$, normalized to $R_{0}^{2} B_{0}$, and the safety factor $q(H)$ on the magnetic surface $H=H_{0}\left(z, p_{z}\right)$ determined from the field line equations are given by

$$
\begin{aligned}
& \psi(H)=\frac{1}{2 \pi} \int_{C} p_{z}(z, H) \mathrm{d} z, \\
& q(H)=\frac{\Delta \varphi}{2 \pi}=\int_{C} \frac{\mathrm{d} z}{\partial H / \partial p_{z}},
\end{aligned}
$$

where the integral is taken along the closed contour $C$ of $H=H_{0}\left(z, p_{z}\right)=$ const. From equations (6) it follows that $H=H(\psi)$ and

$$
\frac{\mathrm{d} H}{\mathrm{~d} \psi}=1 / q(\psi)
$$

The field lines, $z(\varphi), p_{z}(\varphi)$, on each magnetic surface $H$ or $\psi$, can be presented in terms of independent variables $\psi$ and the so-called poloidal angle, $\vartheta$ :

$$
z=z(\psi, \vartheta), \quad p_{z}=p_{z}(\psi, \vartheta),
$$

where $z(\psi, \vartheta), p_{z}(\psi, \vartheta)$ are $2 \pi$-periodic functions of poloidal angle $\vartheta: z(\psi, \vartheta)=z(\psi, \vartheta+2 \pi), p_{z}(\psi, \vartheta)=p_{z}(\psi, \vartheta+2 \pi)$. The poloidal angle $\vartheta$ is a linear function of the toroidal angle $\varphi: \vartheta=\varphi / q(\psi)+\vartheta_{0}$.

According to (2) the $\mathrm{X}$-points $\left(R_{s}, Z_{s}\right)$, i.e. the nulls of the poloidal field $\left(B_{R}, B_{Z}\right)$, on the poloidal section correspond to the hyperbolic fixed points $\left(z_{s}, p_{s}\right)$ of the Hamiltonian system (4) where

$$
\frac{\mathrm{d} z}{\mathrm{~d} \varphi}=\frac{\partial H_{0}}{\partial p_{z}}=0, \quad \frac{\mathrm{d} p_{z}}{\mathrm{~d} \varphi}=-\frac{\partial H_{0}}{\partial z}=0 .
$$

In single-null divertor tokamaks there is only one $\mathrm{X}$-point connecting with itself by the homoclinic orbit on the separatrix.

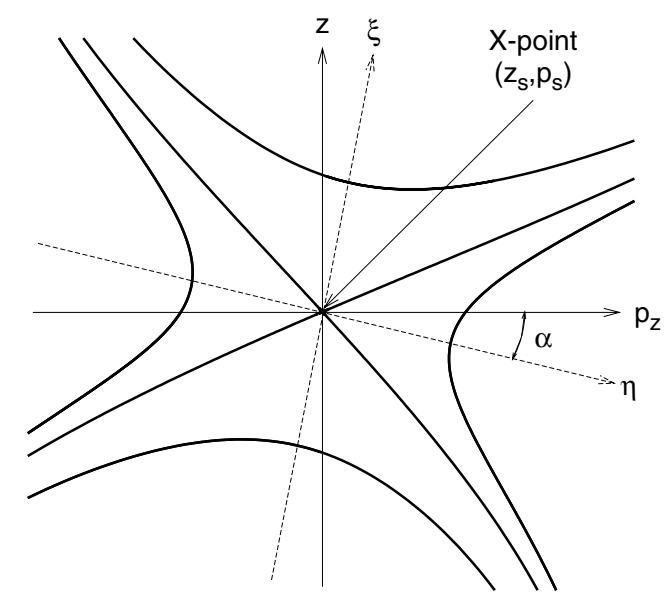

Figure 3. Field lines near the $\mathrm{X}$-point.

In the double-null divertor tokamaks there are two X-points and they are connected with two heteroclinic orbits on the two separatrices, respectively (see figure 1).

Consider field lines near the X-point (see figure 3 ). The unperturbed Hamiltonian $H_{0}\left(z, p_{z}\right)$ can be expanded in a series of powers of $\left(z-z_{s}\right),\left(p_{z}-p_{s}\right)$ near the X-points:

$$
\begin{aligned}
& H_{0}\left(z, p_{z}\right)=H_{0}\left(z_{s}, p_{s}\right)+\frac{1}{2} H_{z z}\left(z-z_{s}\right)^{2} \\
& \quad+H_{z p}\left(z-z_{s}\right)\left(p_{z}-p_{s}\right)+\frac{1}{2} H_{p p}\left(p_{z}-p_{s}\right)^{2} \\
& \quad+O\left[\left(z-z_{s}\right)^{3},\left(p_{z}-p_{s}\right)^{3}\right],
\end{aligned}
$$

where $H_{z z}, H_{z p}$ and $H_{p p}$ are second derivatives of $H_{0}$, i.e. $\partial^{2} H_{0} / \partial z^{2}, \partial^{2} H_{0} / \partial z \partial p, \partial^{2} H_{0} / \partial p^{2}$, taken at the hyperbolic fixed point $z=z_{s}, p=p_{s}$. By the linear transformation of the variable (see figure 3 )

$$
\begin{aligned}
& \xi=\left(z-z_{s}\right) \cos \alpha+\left(p_{z}-p_{s}\right) \sin \alpha, \\
& \eta=-\left(z-z_{s}\right) \sin \alpha+\left(p_{z}-p_{s}\right) \cos \alpha,
\end{aligned}
$$

the Hamiltonian (10) can be diagonalized:

$H_{0}\left(z, p_{z}\right)=H_{0}\left(z_{s}, p_{s}\right)-\frac{\left|\lambda_{1}\right|}{2} \xi^{2}+\frac{\lambda_{2}}{2} \eta^{2}+O\left(\xi^{3}, \eta^{3}\right)$,

where $\lambda_{1}, \lambda_{2}$,

$\left(\lambda_{1}, \lambda_{2}\right)=\frac{H_{z z}+H_{p p}}{2} \pm \sqrt{\frac{\left(H_{z z}-H_{p p}\right)^{2}}{4}+H_{z p}^{2}}$.

$\left(\lambda_{1}<0, \lambda_{2}>0\right)$ are the eigenvalues of the matrix

$$
\left(\begin{array}{ll}
H_{z z} & H_{z p} \\
H_{z p} & H_{p p}
\end{array}\right)
$$

The angle $\alpha$ is determined by $\tan 2 \alpha=H_{z p} /\left(H_{p p}-H_{z z}\right)$.

We introduce the relative poloidal magnetic flux $h$ :

$$
h=H_{0}\left(z, p_{z}\right)-H_{0}\left(z_{s}, p_{s}\right) .
$$

For the closed magnetic field lines the relative flux is negative, $h<0$, and for the open field lines it is positive, $h>0$. At the separatrices, $h=0$ (see figure 1). A behaviour of field lines near the separatrix and the X-points is generic (see, 
e.g. [18]). In particular, the safety factor $q(h)$ has the following asymptotics:

$$
q(h)=\frac{1}{2 \pi \gamma} \ln \frac{Q}{|h|}+O(h), \quad|h| \rightarrow 0,
$$

where $\gamma$ is a parameter determined by the expansion parameter $\lambda_{1}$, and $\lambda_{2}$ in (11): $\gamma=\sqrt{\left|\lambda_{1} \lambda_{2}\right|}$, and $Q$ is a positive constant. Unperturbed field line trajectories lie on the magnetic surfaces of constant $h$ and they can be presented in the form $(R, Z)=R(\varphi ; h), Z(\varphi ; h)$.

The increment of toroidal angle $\Delta \varphi$ along the field lines near the X-point also has universal behaviour. We calculate, for instance, $\Delta \varphi$ along field lines from the axis $\xi$ at $\eta=0$ (or from the axis $\eta$ at $\xi=0$ ) to the line $\eta=$ const ( $\xi=$ const). Using the field line equations near the $\mathrm{X}$-point

$$
\frac{\mathrm{d} \xi}{\mathrm{d} \varphi}=\lambda_{2} \eta, \quad \frac{\mathrm{d} \eta}{\mathrm{d} \varphi}=\lambda_{1} \xi
$$

and the relation $\eta=\sqrt{2 h+\left|\lambda_{1}\right| \xi^{2}} / \sqrt{\lambda_{2}}$, we have

$\Delta \varphi=\frac{1}{\sqrt{\lambda_{2}}} \int_{\xi_{0}}^{\xi} \frac{\mathrm{d} x}{\sqrt{2 h+\left|\lambda_{1}\right| x^{2}}}=\frac{1}{\gamma} \ln \left(\frac{\xi}{b}+\sqrt{\frac{\xi^{2}}{b^{2}}+p}\right)$.

Here $\xi_{0}=0, p=1$ for $h>0$ and $\xi_{0}=b, p=-1$ for $h<0$, where $b=\sqrt{2|h| /\left|\lambda_{1}\right|}$. At the limit $|h| \rightarrow 0$ this integral has the following asymptotics

$$
\Delta \varphi \approx \frac{1}{\gamma} \ln \frac{2 \xi}{\xi_{0}}=\frac{1}{2 \gamma} \ln \frac{2 \lambda_{1} \xi^{2}}{|h|} .
$$

\subsection{Magnetic perturbations and formation of stochastic layer near the separatrix}

Suppose that the magnetic perturbations are described by the toroidal component of the vector potential $A_{\varphi}^{\text {(per) }}(R, Z, \varphi)$ which represented in the form Fourier series:

$A_{\varphi}^{(\text {per })}(R, Z, \varphi)=\epsilon \frac{B_{0} R_{0}^{2}}{R} \sum_{n} A_{n}(R, Z) \cos \left(n \varphi+\chi_{n}\right)$,

where $n$ is the toroidal mode number, $\epsilon=\max \left|A_{\varphi}^{(\text {per })}\right| / B_{0} R_{0}$ is the dimensionless perturbation parameter. Using the expansion (16) we present the perturbed Hamiltonian $H_{1}\left(z, p_{z}, \varphi\right)$ in the form

$$
H_{1}\left(z, p_{z}, \varphi\right)=\sum_{n} H_{n}\left(z, p_{z}\right) \cos \left(n \varphi+\chi_{n}\right),
$$

where $H_{n}\left(z, p_{z}\right)=-A_{n}\left(R\left(p_{z}\right), R_{0} z\right)$.

In the presence of perturbations it is convenient to present the Hamiltonian equations of field lines (4) in the Boozer coordinates, i.e. toroidal flux, $\psi$, and poloidal angle, $\vartheta$ :

$$
\frac{\mathrm{d} \vartheta}{\mathrm{d} \varphi}=\frac{\partial H}{\partial \psi}, \quad \frac{\mathrm{d} \psi}{\mathrm{d} \varphi}=-\frac{\partial H}{\partial \vartheta},
$$

In these coordinates the magnetic field $\boldsymbol{B}$ can be written in the Clebsch form, $\boldsymbol{B}=\nabla \psi \times \nabla \vartheta+\nabla \varphi \times H$. The Hamiltonian can be presented as a sum:

$$
H(\psi, \vartheta, \varphi)=H_{0}(\psi)+\epsilon H_{1}(\psi, \vartheta, \varphi),
$$

where the unperturbed Hamiltonian,

$$
H_{0}(\psi)=\int \frac{\mathrm{d} \psi}{q(\psi)}
$$

describes field lines of equilibrium plasmas, while $\epsilon H_{1}(\psi, \vartheta, \varphi) \equiv$ $H_{1}\left(z(\psi, \vartheta), p_{z}(\psi, \vartheta), \varphi\right)$ corresponds to the perturbation, which can be expanded in Fourier series in the poloidal angle, $\vartheta$, and the toroidal angle, $\varphi$ :

$H_{1}(\psi, \vartheta, \varphi)=\sum_{m n} H_{m n}(\psi) \cos \left(m \vartheta-n \varphi+\chi_{m n}\right)$.

The Fourier components $H_{m n}(\psi)$ correspond to the amplitudes of $(m, n)$-resonant magnetic perturbations which are responsible for the destruction of magnetic surfaces $\psi_{m n}$ with rational values of the safety factor, i.e. $q\left(\psi_{m n}\right)=m / n$, and formation of chain magnetic islands. The width of islands measured in a toroidal flux, $\psi$, is given by (see, e.g. [23])

$$
W_{m n}=4 q\left(\psi_{m n}\right)\left|\frac{\epsilon H_{m n}\left(\psi_{m n}\right)}{\mathrm{d} q / \mathrm{d} \psi}\right|^{1 / 2} .
$$

In the presence of non-axisymmetric perturbations (16) the magnetic separatrices are destroyed [20,23]. The field lines with the initial coordinates located within a certain distance from the separatrix become chaotic. These field lines are not confined in the plasma region, and they leave the plasma region hitting the divertor plates after a certain number of poloidal turns.

The width of the stochastic layer formed near the separatrix can be qualitatively estimated using Chirikov's criteria of overlapping of neighbouring magnetic islands. For this purpose it is convenient to use $h$ variable (13) instead of $\psi$. Resonant magnetic surfaces, $h_{m n}$, defined by $q\left(h_{m n}\right)=m / n$, near the separatrix become dense, i.e. the distance between neighbouring resonant magnetic surfaces, $\delta h=\left|h_{m+1, n}-h_{m n}\right|$, goes to zero with an increase in the poloidal mode number $m$, or equivalently with a decrease in $|h|$. Indeed, using the asymptotics of the safety factor near the separatrix (14) one obtains

$$
\delta h=\frac{2 \pi \gamma}{n}\left|h_{m n}\right|, \quad h_{m n}=-Q \exp \left(-\frac{2 \pi \gamma m}{n}\right)
$$

At the same time the width of the $(m, n)-$ magnetic island in $h$ variable is equal to $\Delta h=W_{m n} / q\left(h_{m n}\right)$, where $W_{m n}$ is defined by equation (22). According to Chirikov (see, [20]) the stochastic motion occurs when the distance between neighbouring islands becomes smaller than widths of islands, i.e.

$$
\sigma(h)=\left(\frac{\Delta h}{\delta h}\right)^{2}=\frac{8 n^{2} \epsilon\left|H_{m n}\right| q(h)}{\pi \gamma|h|} \gtrsim 1 .
$$

Condition (24) is satisfied for the motion near the separatrix with $|h|<h_{c}$, where $h_{c}$ is the boundary value of the stochastic layer determined by the equation, $\sigma\left(h_{c}\right) \sim 1$. A more detailed study of the boundary value will be given in section 4 for the wire model of the plasma.

The quantitative determination of the stochastic layer and its properties can be performed by the integration of field line equation (18). The mapping methods to study this problem will be considered in the next section. 


\section{Mapping approaches}

We describe below two methods to study chaotic field lines near the destroyed separatrix. The first mapping method described in [25-27] allows one to effectively construct Poincaré sections of field lines on poloidal cross sections of the torus. This method has been successfully applied to study the magnetic structure of the dynamic ergodic divertor of the TEXTOR (see [28-32]). The second mapping approach, the separatrix mapping, is the most effective for constructing Poincaré sections on the planes along the toroidal axis which cross the X-points. Both methods are computationally efficient for studying the structure of the stochastic layer and to obtain magnetic footprints on divertor plates.

\subsection{Iterative mapping}

In this mapping method the integration of the Hamiltonian system (18) with a small time-step integration is replaced by return mappings of variables $(\vartheta, \psi)$ to a certain poloidal section of the torus $\varphi=\varphi_{k}$. We define below a mapping of field lines in a toroidal system. Let $\left(\vartheta_{k}, \psi_{k}\right)$ be the poloidal angle and the toroidal flux at the poloidal sections $\varphi=\varphi_{k}=2 \pi k / N$, ( $k=0, \pm 1, \pm 2, \ldots)$, where $N \geqslant 1$ is an integer number. The iterative map is defined as

$$
\left(\vartheta_{k+1}, \psi_{k+1}\right)=\hat{M}\left(\vartheta_{k}, \psi_{k}\right),
$$

which relates the variables $(\vartheta, \psi)$ at the successive sections $\varphi_{k}$ and $\varphi_{k+1}$. Then the map $(\hat{M})^{N}$ defines the Poincaré return map. The flux-preserving property of the mapping (25) is expressed by

$$
\left|\frac{\partial\left(\vartheta_{k+1}, \psi_{k+1}\right)}{\partial\left(\vartheta_{k}, \psi_{k}\right)}\right|=1
$$

The general form of the mapping (25) for the Hamiltonian system (19)-(21) is derived in [26] (see also [27]). In the first order of perturbation amplitude $\epsilon$ it has the following fluxpreserving form:

$\Psi_{k}=\psi_{k}-\epsilon \frac{\partial S^{(k)}}{\partial \vartheta_{k}}, \quad \Theta_{k}=\vartheta_{k}+\epsilon \frac{\partial S^{(k)}}{\partial \Psi_{k}}$,

$\Psi_{k+1}=\Psi_{k}, \quad \bar{\Theta}_{k}=\Theta_{k}+\frac{\varphi_{k+1}-\varphi_{k}}{q\left(\Psi_{k}\right)}$,

$\psi_{k+1}=\Psi_{k+1}+\epsilon \frac{\partial S^{(k+1)}}{\partial \vartheta_{k+1}}, \quad \vartheta_{k+1}=\bar{\Theta}_{k}-\epsilon \frac{\partial S^{(k+1)}}{\partial \Psi_{k+1}}$,

where $S^{(k)} \equiv S\left(\vartheta_{k}, \Psi_{k}\right)$ is the value of the generating function $S\left(\vartheta, \Psi, \varphi, \varphi_{0} ; \epsilon\right)$ taken at sections $\varphi=\varphi_{k}$, i.e. $S\left(\vartheta_{k}, \Psi_{k}\right)=$ $S\left(\vartheta_{k}, \Psi_{k}, \varphi_{k}, \varphi_{0} ; \epsilon\right)$ :

$$
\begin{aligned}
S(\vartheta, & \left.\Psi, \varphi, \varphi_{0}\right)=-\left(\varphi-\varphi_{0}\right) \sum_{m, n} H_{m n}(\Psi) \\
& \times\left[a\left(x_{m n}\right) \sin \left(m \vartheta-n \varphi+\chi_{m n}\right)\right. \\
& \left.+b\left(x_{m n}\right) \cos \left(m \vartheta-n \varphi+\chi_{m n}\right)\right],
\end{aligned}
$$

where

$a(x)=\frac{1-\cos x}{x}, \quad b(x)=\frac{\sin x}{x}$,

$x_{m n}=\left(\frac{m}{q(\Psi)}-n\right)\left(\varphi-\varphi_{0}\right)$.

The free parameter $\varphi_{0}$ lies in the interval $\varphi_{k} \leqslant \varphi_{0} \leqslant \varphi_{k+1}$.
The mapping method requires the knowledge of the safety factor $q(\psi)$ and Fourier coefficients $H_{m n}(\psi)$ of the perturbation Hamiltonian (21). In realistic magnetic configurations analytical determination of these functions is extremely difficult. For this reason we have used the following method of numerical interpolations of these quantities from the pre-calculated values of $q(\psi)$ and $H_{m n}(\psi)$ on a grid of magnetic fluxes $\psi: \psi_{i}=i \Delta \psi,\left(i=1, \ldots, N_{\psi}\right)$ by the numerical integration of the equations of field lines (4). To evaluate the functions $q(\psi), H_{m n}(\psi)$ for the arbitrary values of $\psi$ the cubic splines of their pre-calculated values $q\left(\psi_{i}\right)$, $H_{m n}\left(\psi_{i}\right)$ are employed.

In order to display the orbits in real space coordinates $\boldsymbol{x}=(R, Z)$ the relations of these coordinates with the variables $(\psi, \vartheta)$ are also found by the numerical integration of the equations of field lines in the absence of magnetic perturbations. These relationships are presented in the form of Fourier series

$z=\sum_{m}\left(z_{m}^{(s)}(\psi) \sin m \vartheta+z_{m}^{(c)}(\psi) \cos m \vartheta\right)$,

$p_{z}=\sum_{m}\left(p_{z m}^{(s)}(\psi) \sin m \vartheta+p_{z m}^{(c)}(\psi) \cos m \vartheta\right)$

The Fourier coefficients $z_{m}^{(s)}(\psi), \ldots, p_{m}^{(c)}(\psi)$ and $\alpha_{m}(\psi)$ can also be calculated numerically for the same grid coordinates of $\psi$, and their values for arbitrary $\psi$ are interpolated by the cubic splines.

This mapping method will be applied to study field lines near the separatrix for the three-wire model in section 4 .

\subsection{Separatrix map}

In this section we describe another method of construction of mappings near the separatrix proposed in [21,22]. Let $\left(\varphi_{k}, h_{k}\right)$ be values of the toroidal angle, $\varphi$, and the relative poloidal flux, $h$, at the crossing point of field line with a certain toroidal section $\Sigma$. It is convenient to define these sections $\left(\Sigma_{s}\right)$ near the X-points where field lines stay longer. In order to determine magnetic footprints we should also construct mappings to sections coinciding with the divertor plates $\left(\Sigma_{d}\right)$. The locations of these sections on the poloidal plane and the magnetic configuration of systems in the absence of the divertor plates are shown in figure 4 in the case of the singlenull divertor tokamak. In this case there is one $\mathrm{X}$-point and two homoclinic saddle-saddle connections, $C_{p}$ and $C_{c}$, on the plasma and coil regions, respectively (see figure 4).

According to definitions given in [21], the cross section $\Sigma_{s}$ consists of two stripes (segments in the poloidal $(R, Z)$ plane) along the $\xi$ and $\eta$ axes transversely crossing each other along the $\mathrm{X}$-line (at the $\mathrm{X}$-point on the $(R, Z)$-plane). The unperturbed field lines cross these stripes transversely (see figure 3).

We study the perturbed field lines near the separatrix. Let $\left(\varphi_{k}, h_{k}\right)$ be the toroidal angle, $\varphi$ and the poloidal flux, $h$, at the $k$ th crossing point of the field lines with the cross section $\Sigma_{s}$. Our aim is to construct the mapping

$$
\left(\varphi_{k+1}, h_{k+1}\right)=\hat{M}\left(\varphi_{k}, h_{k}\right),
$$

connecting two consecutive crossing points of field line at the $\operatorname{section}(\mathrm{s}) \Sigma_{s}$ 


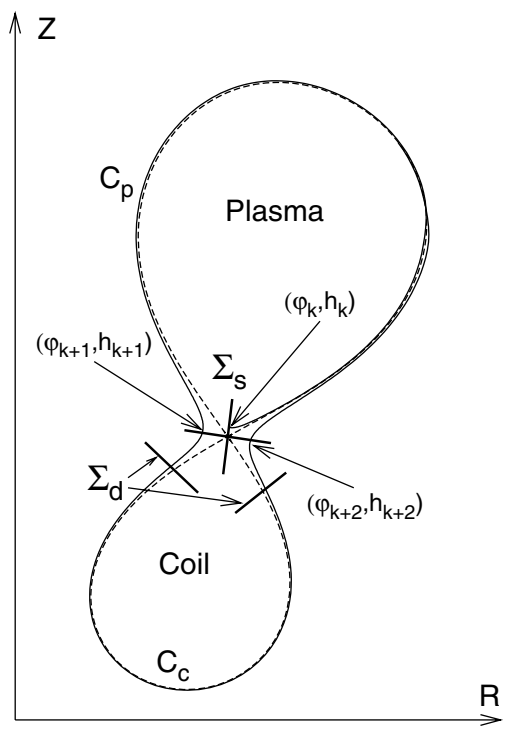

Figure 4. Geometry of the separatrix map in a single null poloidal divertor tokamak. The solid curves describe perturbed field lines and the dashed curves describe the unperturbed separatrix.

In the case of the single-null divertor there are two mappings $\hat{M}^{(j)}, j=(p, c)$ (30) along the separatrices $C_{p}$ and $C_{c}$ in the plasma and coil regions, respectively, which completely determine the dynamics of the perturbed field lines near the separatrix (see figure 4)).

According to results obtained in [21] for the Hamiltonian system $H_{0}\left(z, p_{z}\right)$ in the presence of perturbation (17) the mapping along the $j$ th saddle-saddle connection is given by

$h_{k+1}=h_{k} \mp \epsilon F^{( \pm)}\left(\varphi_{k}, h_{k+1}, h_{k}\right)$,

$\varphi_{k+1}=\varphi_{k} \pm \pi\left[q^{(j)}\left(h_{k}\right)+q^{(j)}\left(h_{k+1}\right)\right] \pm \epsilon G^{( \pm)}\left(\varphi_{k}, h_{k+1}, h_{k}\right)$,

where

$$
\begin{aligned}
& F^{( \pm)}\left(\varphi_{k}, h_{k+1}, h_{k}\right)=\sum_{n} n\left(K_{n}\left(h_{k+1}\right) \sin \Phi_{n}^{( \pm)}\left(\varphi_{k}, h_{k}\right)\right. \\
& \left.\quad+L_{n}\left(h_{k}\right) \cos \Phi_{n}^{( \pm)}\left(\varphi_{k}, h_{k}\right)\right), \\
& G^{( \pm)}\left(\varphi_{k}, h_{k+1}, h_{k}\right)=-\sum_{n}\left(\frac{\mathrm{d} K_{n}\left(h_{k+1}\right)}{\mathrm{d} h_{k+1}} \cos \Phi_{n}^{( \pm)}\left(\varphi_{k}, h_{k}\right)\right. \\
& \left.\quad-\frac{\mathrm{d} L_{n}\left(h_{k+1}\right)}{\mathrm{d} h_{k+1}} \sin \Phi_{n}^{( \pm)}\left(\varphi_{k}, h_{k}\right)\right),
\end{aligned}
$$

$\Phi_{n}^{( \pm)}\left(\varphi_{k}, h_{k}\right)=n\left(\varphi_{k} \pm \pi q^{(j)}\left(h_{k}\right)\right)+\chi_{n}$

In equations (31)-(33) the positive sign $(+)$ corresponds to the forward map, i.e. along the positive direction of the toroidal angle $\varphi$, and the negative sign (-) corresponds to the backward map, i.e. along the negative direction of the toroidal angle $\varphi$. The mapping (31) is a flux-preserving, i.e. $\operatorname{det}\left|\partial\left(h_{k+1}, \varphi_{k+1}\right) / \partial\left(h_{k}, \varphi_{k}\right)\right|=1$. It is also invariant with respect to the time reversing transformation, $k \leftrightarrow k+1$.

The effective safety factor $q^{(j)}(h)$ near the $j$ th saddlesaddle connection is given by

$$
q^{(j)}(h)=\frac{1}{2 \pi} \int_{C^{(j)}} \frac{\mathrm{d} z}{\partial H_{0} / \partial p_{z}},
$$

where the integral is taken along the contour $C^{(j)}$ of $H=$ $H_{0}\left(z, p_{z}\right)=$ const connecting the sections $\Sigma_{s}$. In the singlenull divertor and in the coil regions in the double-null divertor it coincides with the safety factor (6). Near the separatrix the safety factors $q^{(j)}(h)$ have the universal asymptotics of type (14) with the same parameter $\gamma$ but different constant parameters $Q=Q_{j}$ corresponding to the different contours $C^{(j)}$.

The coefficients $K_{n}(h), L_{n}(h)$ are the Melnikov type integrals

$R_{n}(h)=K_{n}(h)+\mathrm{i} L_{n}(h)=\int_{-\pi q(h)}^{\pi q(h)} V_{n}(h, \tau) \mathrm{e}^{\mathrm{i} n \tau} \mathrm{d} \tau$,

taken over the functions $V_{n}^{(j)}(h, \tau) \equiv H_{n}\left(z^{(j)}\left(h, \varphi-\varphi_{0}\right)\right.$, $\left.p_{z}^{(j)}\left(h, \varphi-\varphi_{0}\right)\right)$ along the unperturbed field lines near the $j$ th separatrix. At $h=0$ the integral is taken along the unperturbed separatrix and it corresponds to the Melnikov integral [24].

As was shown in [22] the integrals $R_{n}(h)$ can be presented as a sum of regular, $R_{n}^{(\mathrm{reg})}(h)$, and oscillatory, $R_{n}^{(\mathrm{osc})}(h)$, parts, i.e.

$$
R_{n}(h)=R_{n}^{(\mathrm{reg})}(h)+R_{n}^{(\mathrm{osc})}(h) .
$$

The regular part, $R_{n}^{(\mathrm{reg})}(h)$, is a smooth function of the relative poloidal flux $h$. The oscillatory part, $R_{n}^{(\text {osc })}(h)$, is a rapidly oscillating function of $h$, with a local period of oscillations. The zeros of $R_{n}^{(\mathrm{reg})}(h)$, i.e. $R_{n}^{(\mathrm{reg})}\left(h_{m n}\right)=0$, coincide with the resonant poloidal fluxes of primary resonances, $q\left(h_{m n}\right)=$ $m / n$. Since field lines are mostly affected near the primary resonances where oscillatory terms of the integrals $R_{n}(h)$ vanish, we can retain only the smooth regular parts $R_{n}^{(\text {reg })}(h)$ (see [22]).

Further for the very thin stochastic layer near the separatrix the mapping (31) can be simplified by replacing the integrals $K_{n}(h), L_{n}(h)$ by their values $K_{n}(0), L_{n}(0)$ at the separatrix $h=0$.

$$
\begin{aligned}
& K_{n}^{(j)}(0)=\int_{-\infty}^{\infty} V_{n}^{(j)}(0, \tau) \cos (n \tau) \mathrm{d} \tau, \\
& L_{n}^{(j)}(0)=\int_{-\infty}^{\infty} V_{n}^{(j)}(0, \tau) \sin (n \tau) \mathrm{d} \tau .
\end{aligned}
$$

Then we obtain the mapping along the $j$ th saddle-saddle connection:

$$
\begin{aligned}
& h_{k+1}=h_{k} \mp \epsilon \sum_{n} n\left(K_{n}^{(j)}(0) \sin \left[n\left(\varphi_{k} \pm \pi q^{(j)}\left(h_{k}\right)\right)+\chi_{n}\right]\right. \\
& \left.\quad+L_{n}^{(j)}(0) \cos \left[n\left(\varphi_{k} \pm \pi q^{(j)}\left(h_{k}\right)\right)+\chi_{n}\right]\right),
\end{aligned}
$$

$\varphi_{k+1}=\varphi_{k} \pm \pi\left[q^{(j)}\left(h_{k}\right)+q^{(j)}\left(h_{k+1}\right)\right]$.

The set of mappings $\hat{M}^{(j)}$ given by equations (31) and (38) completely describe perturbed field lines near the separatrices of magnetic system. They are determined only by the safety factors $q^{(j)}(h)(34)$ and the Melnikov type integrals $K_{n}^{(j)}(h)$, $L_{n}^{(j)}(h)(35),(37)$.

\subsection{Mappings to the divertor plates}

To determine magnetic footprints on divertor plates we need to construct the mapping of field lines from sections $\Sigma_{s}$ to the 
divertor sections $\Sigma_{d}$. Since these sections are located close to the $X$-point it takes for field lines less than half of the poloidal turns to reach the divertor plate from section $\Sigma_{s}$. These field lines can be traced along unperturbed field lines starting from its coordinates $\left(\varphi_{k}, h_{k}\right)$ at $\Sigma_{s}$ until they reach the divertor plate $\Sigma_{d}$. Let $\left(\varphi_{d}, h_{d}\right)$ be the toroidal angle and the relative poloidal on the divertor plate. Then the map $\left(\varphi_{k}, h_{k}\right) \rightarrow\left(\varphi_{d}, h_{d}\right)$ is given by

$$
h_{d}=h_{k}, \quad \varphi_{d}=\varphi_{k}+\Delta \varphi\left(h_{k}\right),
$$

where $\Delta \varphi(h)$ is the increment of the toroidal angle $\varphi$ necessary to reach the plate $\Sigma_{d}$ along the unperturbed field lines on the magnetic surface of constant $h=h\left(z, p_{z}\right)$. If the divertor plate $\Sigma_{d}$ is sufficiently close to the X-point the quantity $\Delta \varphi(h)$ can be estimated by equation (15).

Magnetic footprints on divertor plates are obtained by tracing field lines by the mappings (31), (38) and (39) using the following procedure. We choose a set of field lines inside plasma with coordinates $\left(\varphi_{0}, h_{0}\right)$ at section $\Sigma_{s}$. The relative poloidal flux $h$ inside the plasma is negative, $h<0$. We apply the forward and backward mappings (31), (38) in the plasma region until field lines cross the unperturbed separatrix, when $h_{k}>0$. Then using the map (39) we find the coordinates $\left(\varphi_{d}, h_{d}\right)$ on the divertor plate.

\subsection{The accuracy of the mappings}

The iterative mapping given by equation (27) is obtained by retaining the first term in an expansion series of the generating function in powers of the small parameter $\mu=\epsilon(\Delta \varphi)^{v}$, where $\Delta \varphi=\varphi_{k+1}-\varphi_{k}$ is a map step in the toroidal angle, and $v \geqslant 1$ is a constant (see [26]). Therefore, the accuracy of this mapping depends not only on the dimensionless perturbation parameter, $\epsilon$, but also on the mapping step $\Delta \varphi$. As was shown in [26] the mapping with the step comparable with the period of perturbation field, $2 \pi / n$, has the same accuracy as a standard integrator of ordinary differential equations, for instance the Runge-Kutta method, with the integration steps two or three orders smaller. The desired accuracy of the mapping can be controlled by the change of the mapping step $\Delta \varphi$.

The accuracy of the separatrix mapping (equations (31) and (38)) is less studied than the one for the iterative mapping. It is also obtained by retaining the first order term in the expansion of the generating function in powers of $\epsilon$, i.e. the separatrix mapping is valid for small perturbation parameter, $\epsilon \ll 1$. However, the step of the mapping is one poloidal turn which corresponds to the large increment in the toroidal angle, $\Delta \varphi=2 \pi q(h)$. Therefore, this mapping is less accurate than the iterative mapping. A comparison with the standard numerical integration shows that the separatrix mapping well reproduces the structure of the stochastic layer near the separatrix, i.e. the positions of magnetic islands and their sizes. Typically, the accuracy of the mapping is about several per cent for the perturbation parameter $\epsilon \sim 10^{-2}$ (see, also an example in [22]).

\section{Three-wire model of the plasma}

\subsection{Model of equilibrium plasma}

To demonstrate the described method of the separatrix mapping we consider the simple model of the tokamak plasma. The

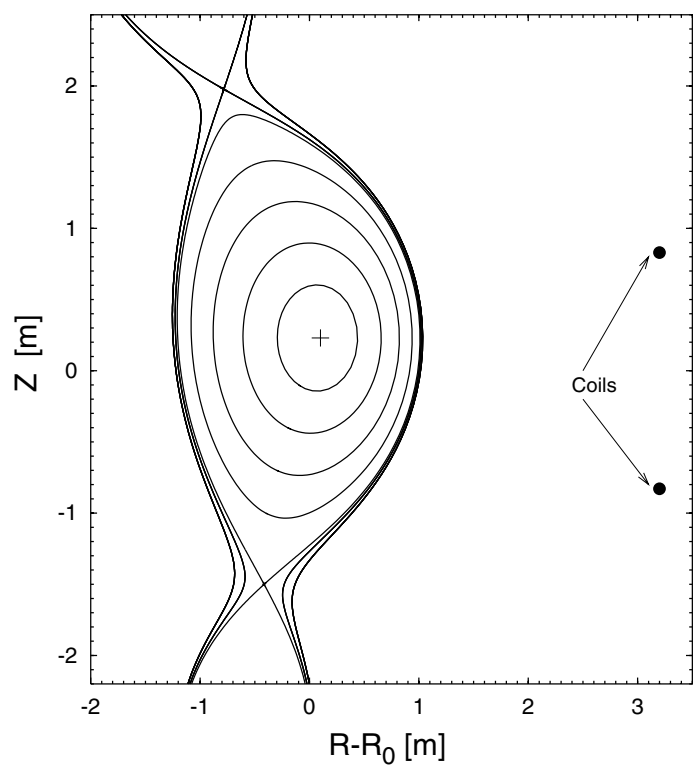

Figure 5. Magnetic configuration of system. Parameters: $B_{0}=2 \mathrm{~T}$, $R_{0}=3 \mathrm{~m}, I_{p} \equiv I_{2}=2 \mathrm{MA}, I_{1}=2 \mathrm{MA}$ and $I_{3}=2.59 \mathrm{MA}$. Coordinates of currents $\left(R_{1}, Z_{1}\right)=(2.4,-2.77) \mathrm{m}$, $\left(R_{2}, Z_{2}\right)=(3.1,0.23) \mathrm{m}$ and $\left(R_{3}, Z_{3}\right)=(1.6,3.7) \mathrm{m}$. The lower $\mathrm{X}$-point is located at $\left(X_{s}, Z_{s}\right)=(-0.4149,-1.5004) \mathrm{m}$.

model consists of three current loops (wires) located at $R_{i}, Z_{i}$, $(i=1,2,3)$, carrying the currents $I_{1}, I_{2}$ and $I_{3}$, respectively. The second loop corresponds to the plasma current $I_{p}$. Such a model of the plasma can describe a single-null and doublenull divertor tokamak plasma configuration near the separatrix which do not significantly depend on the radial profiles of the plasma current $I_{p}$. The example of closed and open magnetic surfaces for this model in the absence of magnetic perturbations is shown in figure 5 by dashed curves.

The vector potential $A_{\varphi}(R, Z)$ of each current loop is given by (see [33])

$A_{\varphi}(R, Z)=\sum_{j=1}^{3} \frac{\mu_{o} I_{j}}{\pi k_{j}} \sqrt{\frac{R_{0}}{R}}\left[\left(1-\frac{k_{j}^{2}}{2}\right) K\left(k_{j}\right)-E\left(k_{j}\right)\right]$,

where $K(k)$ and $E(k)$ are the complete elliptic integrals with the module

$k_{j}^{2}=\frac{4 R_{0} R}{\left(R+R_{0}\right)^{2}+\left(Z-Z_{j}\right)^{2}}, \quad j=1,2,3$.

For large aspect ratio tokamaks $R_{0} / a \gg 1,\left|1-k^{2}\right| \ll 1$ and for the toroidal field $B_{\varphi}(R)=B_{0} R_{0} / R$ the unperturbed Hamiltonian $H_{0}\left(z, p_{z}\right)=-R A_{\varphi}(R, Z) / B_{0} R_{0}^{2}$ up to constant terms can be approximated by

$$
H_{0}\left(z, p_{z}\right)=-R_{0}^{-1} \sum_{j=1}^{3} L_{j}\left(\ln \frac{64}{r_{j}^{2}}-4\right),
$$

where

$$
\begin{aligned}
& r_{j}^{2}=\left(x-x_{j}\right)^{2}+\left(z-z_{j}\right)^{2}, \\
& x_{j}=R_{j} / R_{0}-1, \quad z_{j}=Z_{j} / R_{0}, \\
& L_{j}=\mu_{o} I_{j} / 4 \pi B_{0}, \quad(j=1,2,3) .
\end{aligned}
$$

The quantity $L_{j}$ is the length scale. 


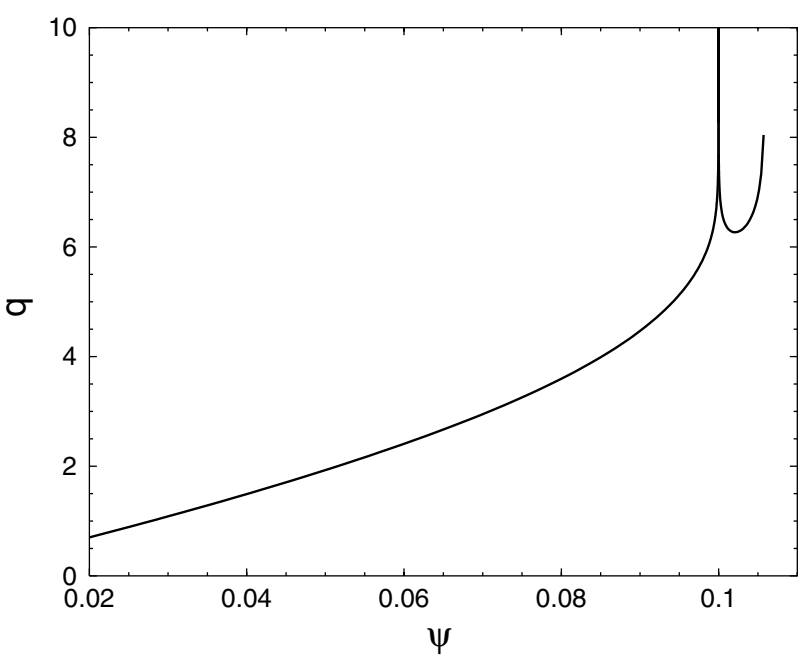

Figure 6. Safety factor $q(\psi)$ profile versus toroidal flux $\psi$. The value of $\psi$ at the separatrix is $\psi_{a}=0.1$.

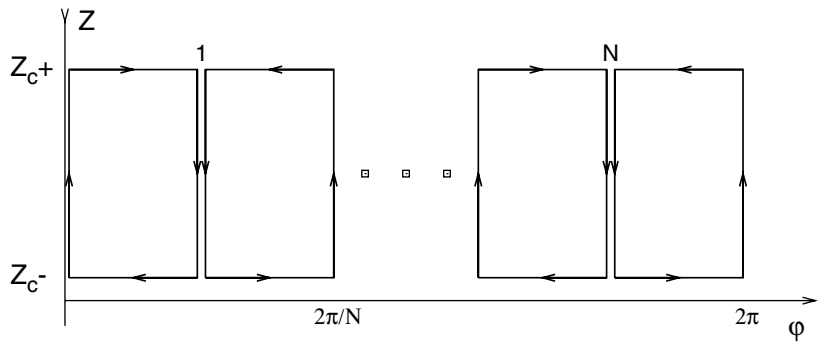

Figure 7. Schematic view of perturbation current loops in the $(\varphi, Z)$ plane.

The magnetic configuration of system and the safety factor profile are shown in figures 5 and 6 for the following parameters of the JET tokamak: a major radius $R_{0}=3 \mathrm{~m}$, a toroidal field at the magnetic axis $B_{0}=2 \mathrm{~T}$. Magnetic axis is located at the position of the second wire $\left(R_{2}, Z_{2}\right)=$ $(3.1,0.23) \mathrm{m}$ with the plasma current $I_{p} \equiv I_{2}=2 \mathrm{MA}$. For the positions of the first, $\left(R_{1}, Z_{1}\right)=(2.4,-2.77) \mathrm{m}$, and third, $\left(R_{3}, Z_{3}\right)=(1.6,3.7) \mathrm{m}$, coils and their currents $I_{1}=2 \mathrm{MA}$ and $I_{3}=2.59 \mathrm{MA}$ are chosen with such values in order to have a single-null divertor configuration with the lower X-point at $\left(X_{s}, Z_{s}\right)=(-0.4149,-1.5004) \mathrm{m}$.

The parameter $\gamma$ in the asymptotic formula (14) for the safety factor $q(h)$ has an order of $\gamma \sim 4 L_{p} R_{0} / a^{2}$, where $L_{p}=\mu_{o} I_{p} / 4 \pi B_{0}$ is the length scale and $a$ is the minor radius. For the above mentioned plasma parameters its exact value is 0.44174 . The parameter $Q$ found by fitting this formula with the numerically calculated $q(h)$ is equal to $Q=5389.63$ for $h<0$ (or $\left.\psi<\psi_{a}\right)$ and $Q=4352.13$ for $h>0\left(\psi>\psi_{a}\right)$.

\subsection{Magnetic perturbations}

Suppose that magnetic perturbations are created by $N$ pair of loop coils with opposite flowing currents $\pm I_{c}$. This is shown schematically in figure 7. The horizontal coordinates of the coils are located at the positions $\left(R_{c+}, Z_{c+}\right)$ and $\left(R_{c-}, Z_{c-}\right)$, respectively. The coordinates $\left(R_{c \pm}, Z_{c \pm}\right)$ do not vary along the toroidal angle $\varphi$. The magnetic field created by these coils is mainly determined by the toroidal component of the vector potential $A_{\varphi}^{(\text {per })}$ and is given by an equation such as equation (40). Then for the large aspect ratio case the perturbation the Hamitonian $H_{1}\left(z, p_{z}, \varphi\right)$ can be approximated by the Hamiltonian

$$
\epsilon H_{1}\left(z, p_{z}, \varphi\right)=L_{c}(\varphi) R_{0}^{-1}\left(\ln r_{c+}^{2}-\ln r_{c-}^{2}\right),
$$

where $L_{c}(\varphi)=\mu_{o} I_{c}(\varphi) / 4 \pi B_{0}$ and

$$
\left.r_{c \pm}^{2}=\left(x-x_{c \pm}\right)^{2}+\left(z-z_{c \pm}\right)\right)^{2} .
$$

In equation (43) the toroidal component of the current $I_{c}(\varphi)$ is periodically changing along the toroidal angle $\varphi$. We present $I_{c}(\varphi)$ by the following discontinuous function of $\varphi$ :

$I_{c}(\varphi)=(-1)^{k} I_{c}, \quad$ for $(\pi / N) k<\varphi<(\pi / N)(k+1)$,

$k=0, \ldots, 2 N-1$.

where $2 N$ is the number of current loops. It can be expanded into a Fourier series

$$
I_{c}(\varphi)=\frac{4 I_{c}}{\pi} \sum_{p=0}^{\infty} \frac{\sin [(2 p+1) N \varphi]}{2 p+1} .
$$

Then the perturbation Hamiltonian is reduced to

$$
\begin{aligned}
& \epsilon H_{1}\left(z, p_{z}, \varphi\right)=\epsilon \sum_{p=0}^{\infty} \frac{1}{2 p+1} H_{N}\left(z, p_{z}\right) \sin [(2 p+1) N \varphi], \\
& H_{N}\left(z, p_{z}\right)=\frac{4 L_{p}}{\pi R_{0}}\left(\ln r_{c+}^{2}-\ln r_{c-}^{2}\right) .
\end{aligned}
$$

The perturbation parameter $\epsilon$ is defined as $\epsilon=I_{c} / I_{p}$.

Furthermore, we retain in the perturbing Hamiltonian (46) only the first term $p=0$, since, as we will see later, this term gives the main effect of the magnetic perturbation since the contribution from higher toroidal modes $n=(2 p+1) N$, $(p \neq 0)$, exponentially decreases with increasing $n$ (see figure 11).

The spectrum of modes $H_{m n}(\psi)$ at different rational magnetic surfaces is shown in figure $8(a)$. Figure $8(b)$ shows the dependence of several modes $H_{m}(\psi)$ on the toroidal flux coordinate $\psi$ (normalized to the maximum flux $\psi_{a}=0.1$ ). The dependencies of the Melnikov type integrals, $K_{n}(h)$, $L_{n}(h)$ (see equation (35)) on the relative poloidal flux, $h$, for the toroidal mode $n=4$ are shown in figure 9. The plot of the module, $\left|R_{n}(h)\right|$, and the phase, $\chi_{n}(h)$, of the complex Melnikov type integral, $R_{n}(h)=K_{n}(h)+\mathrm{i} L_{n}(h)$, versus $h$ is given in figure 10.

The strength of perturbation in the mappings (31), (38) is determined by the module of integrals $K_{n}(h), L_{n}$, i.e. $R_{n}(h)=\sqrt{K_{n}^{2}(h)+L_{n}^{2}(h)}$. The dependence of this quantity at the value $h=0$ on the toroidal mode number $n$ is presented in figure 11. As seen from the figure the effect of the perturbation exponentially decreases with an increase in the toroidal mode $n$.

One can show that at the resonant value of $h=h_{m n}$, i.e. $q\left(h_{m n}\right)=m / n$, the integral $R_{n}(h)$ is related to the spectral components $H_{m n}(h)$,

$H_{m n}\left(h_{m n}\right)=\frac{1}{2 \pi q\left(h_{m n}\right)} R_{n}^{*}\left(h_{m n}\right)=\frac{n}{2 \pi m} R_{n}^{*}\left(h_{m n}\right)$. 

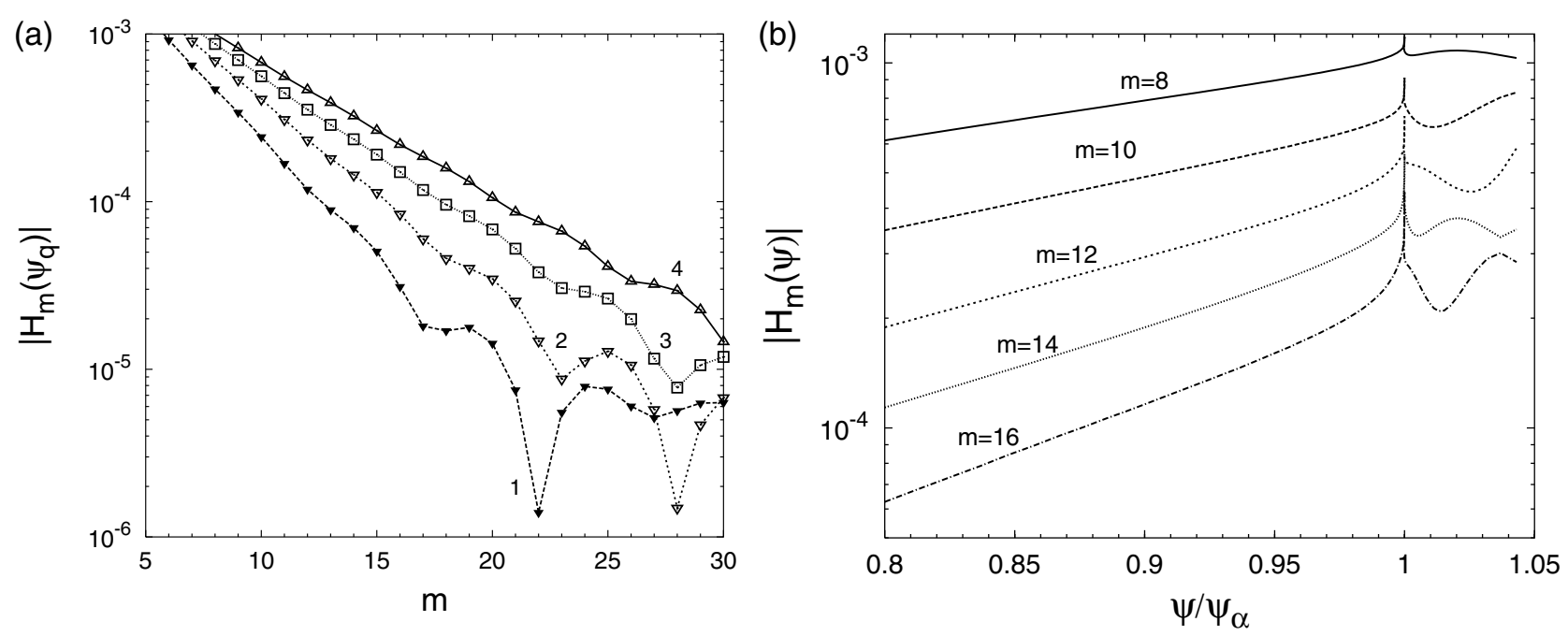

Figure 8. (a) Spectrum of magnetic perturbations $\left|H_{m n}(\psi)\right|$, at the several rational magnetic surfaces: curve 1 corresponds to $q=3$, curve $2-q=4$, curve $3-q=5$ and curve $4-q=6$. $(b)$ Dependence of mode components $\left|H_{m n}(\psi)\right|$ on the normalized toroidal flux $\psi / \psi_{a}$ for the several poloidal mode numbers $m$. The toroidal mode $n=4$ is fixed.

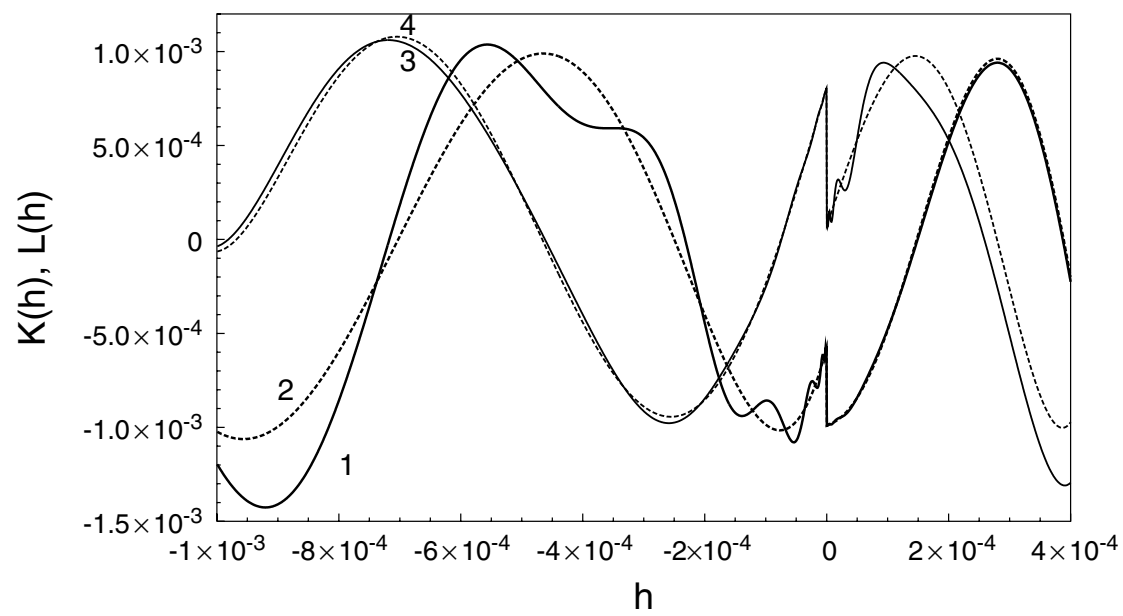

Figure 9. Dependences of the Melnikov type integrals $K_{n}(h)$ (curve 1) and $L_{n}(h)$ (curve 2) on the relative poloidal flux $h$. Curves 3 and 4 correspond to the regular parts of these integrals $K_{n}^{\text {(reg) }}(h)$ and $L_{n}^{(\mathrm{reg})}(h)$, respectively. The toroidal mode number $n=4$.

Moreover, at the resonant value of $h$ the integral $R_{n}(h)$ is determined only by its regular part $R_{n}^{(\mathrm{reg})}(h)$ since the oscillatory part $R_{n}^{\text {(osc) }}(h)$ has zeros at $h=h_{m n}$.

The relation (47) allows one to express equation (24) for the Chirikov criteria of overlapping resonances in the case of perturbation with a single toroidal mode $n$ through only one function $R_{n}^{(\mathrm{reg})}(h)$ :

$$
\sigma(h)=\frac{4 n^{2}}{\pi^{2}} \frac{\epsilon\left|R_{n}^{(\mathrm{reg})}(h)\right|}{\gamma|h|} \gtrsim 1 .
$$

equation (48) can be used for the qualitative estimation of the boundary of the stochastic layer $h_{c}$ for the small values of perturbation $\epsilon \ll 1$. Since $R_{n}^{(\mathrm{reg})}(h) \approx R_{n}^{(\mathrm{reg})}(0)$ we have

$$
\left|h_{c}\right| \sim \frac{4 n^{2}}{\pi^{2}} \frac{\epsilon\left|R_{n}^{(\mathrm{reg})}(0)\right|}{\gamma} .
$$

This estimation for the boundary of the stochastic layer coincides with the one obtained in [19] using the stretching condition of an infinitesimal phase interval (see [34]).
From equation (49) and figure 11 it follows that the width of the stochastic layer $h_{c}$ has the following dependence of $n:\left|h_{c}\right| \sim n^{2} \exp (-A n)$ where $A \approx 1.1$ is a constant independent of $n$. The maximum width $h_{c}$ is achieved at $n=n_{m}=2 / A \approx 2, h_{c}$ decreases with increasing the toroidal mode number $n>n_{m}$. The value of $h_{c}$ also strongly depends on the safety factor profile, $q$, through the parameter $\gamma$ which, according to (14), determines the asymptotics of the $q$-profile near the separatrix. And finally, $h_{c}$, grows linearly with the perturbation amplitude $\epsilon$.

\subsection{The structure of the stochastic layer}

First we describe the structure of the stochastic layer near the magnetic separatrix obtained using the iterative mapping (27). Consider the two kinds of Poincaré sections of field lines. The first one is a mapping of field lines to the polidal section $\varphi=$ constant after each full toroidal turn. The second one is to map the field lines to the plane parallel to the toroidal direction 


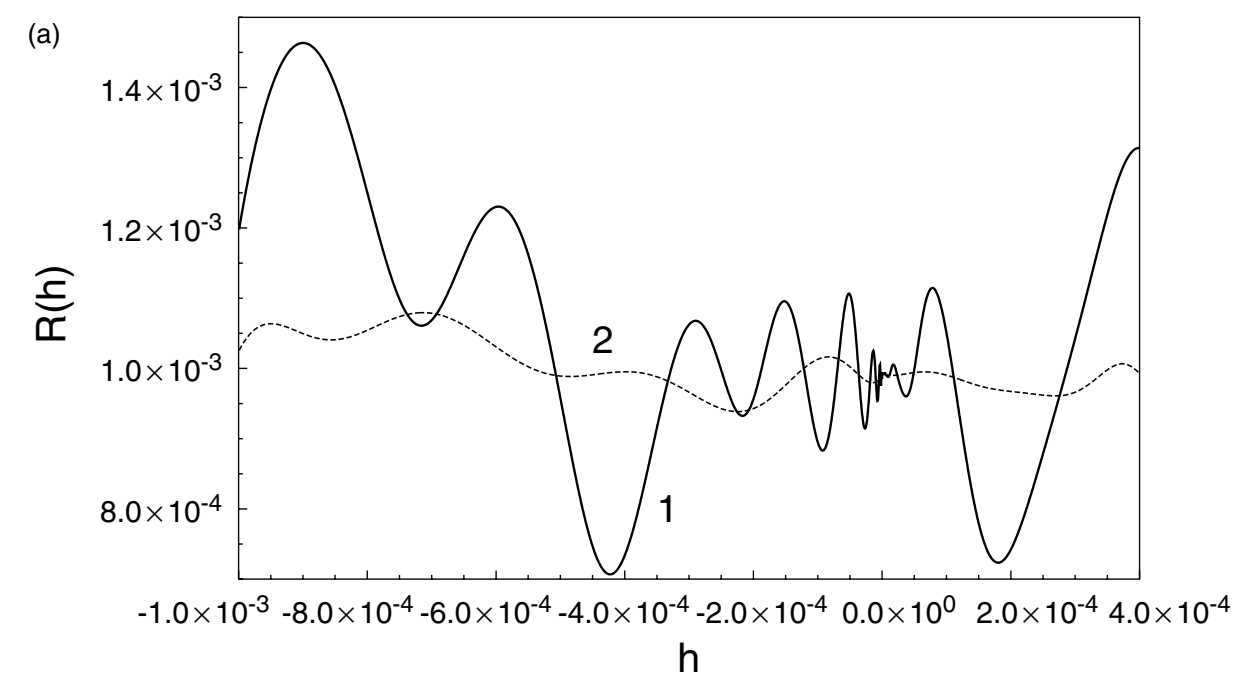

(b)

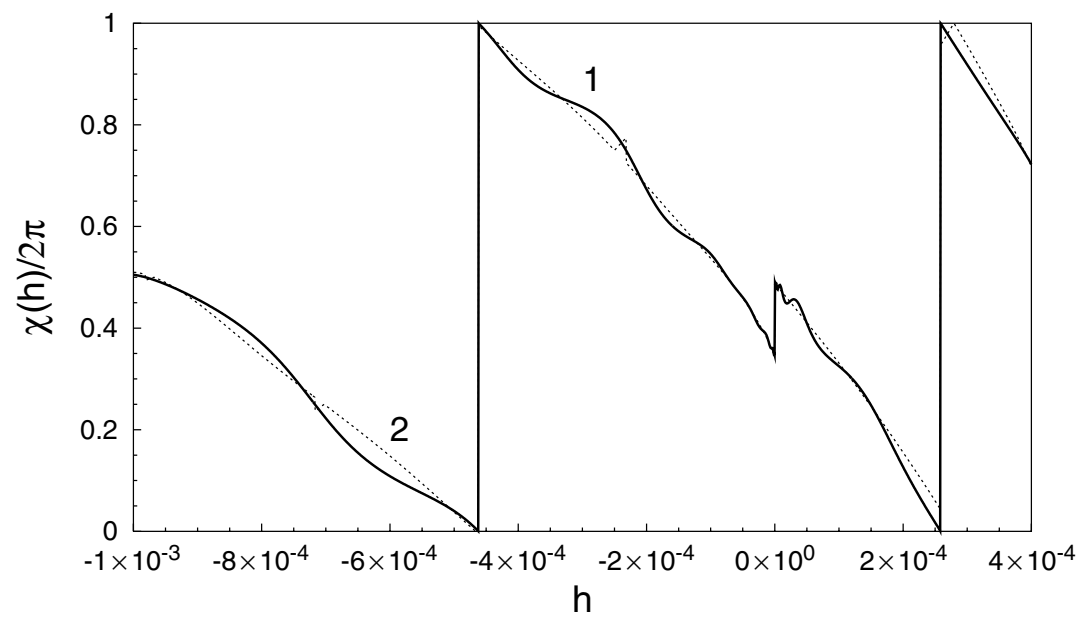

Figure 10. Melnikov type integral $R_{n}(h)=K_{n}(h)+\mathrm{i} L_{n}(h)=\left|R_{n}(h)\right| \mathrm{e}^{\mathrm{i} \chi(h)}$ versus the relative poloidal flux $h .(a)$ Module $\left|R_{n}(h)\right|$ (curve 1) versus $h$ and $\left|R_{n}^{\text {(reg) }}(h)\right|$ (curve 2). (b) Phases $\chi(h)$ (curve 1) $\chi^{\text {(reg) }}(h)$ versus $h$.

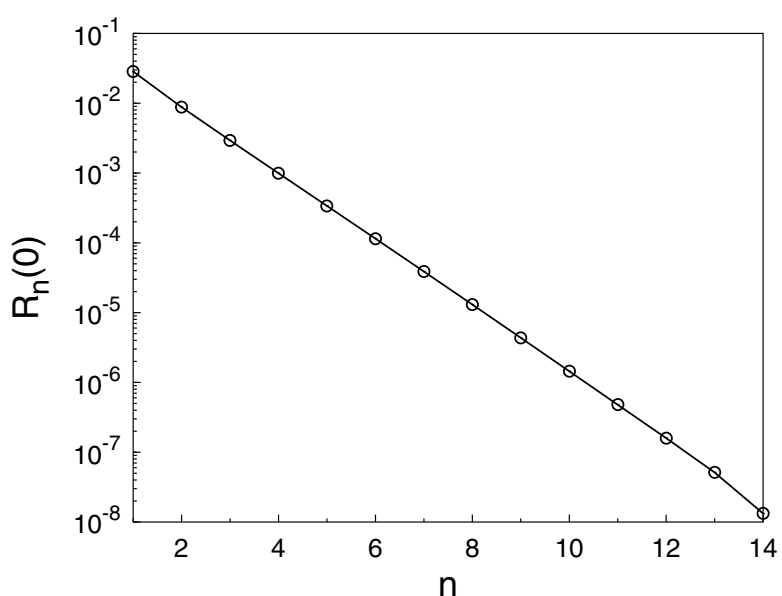

Figure 11. Module $R_{n}(0)=\sqrt{K_{n}^{2}(0)+L_{n}^{2}(0)}$ of the Melnikov type integrals at the separatrix $h=0$ versus the toroidal mode number $n$.

after one full poloidal turn. The schematic view of the mapping of field lines to this kind of section is shown in figure 12 . We map the field lines to the plane $\left(\varphi, X=R_{0} \xi\right)(\xi>0)$, where the coordinate $X$ coincides with the upper branch

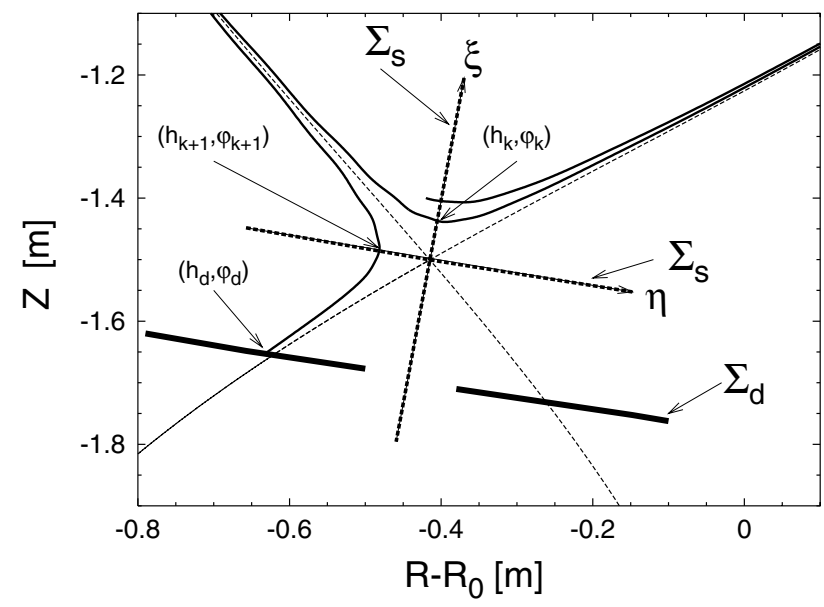

Figure 12. Scheme of mapping of field lines to the section $\Sigma_{s}$ and to the divertor plates.

of the section $\Sigma_{s}$. Poincaré sections presented below are obtained by iterating mapping (27) along the positive direction of the toroidal angle $\varphi$. One can also construct Poincaré sections by iterating the mapping in the negative direction of $\varphi$. 

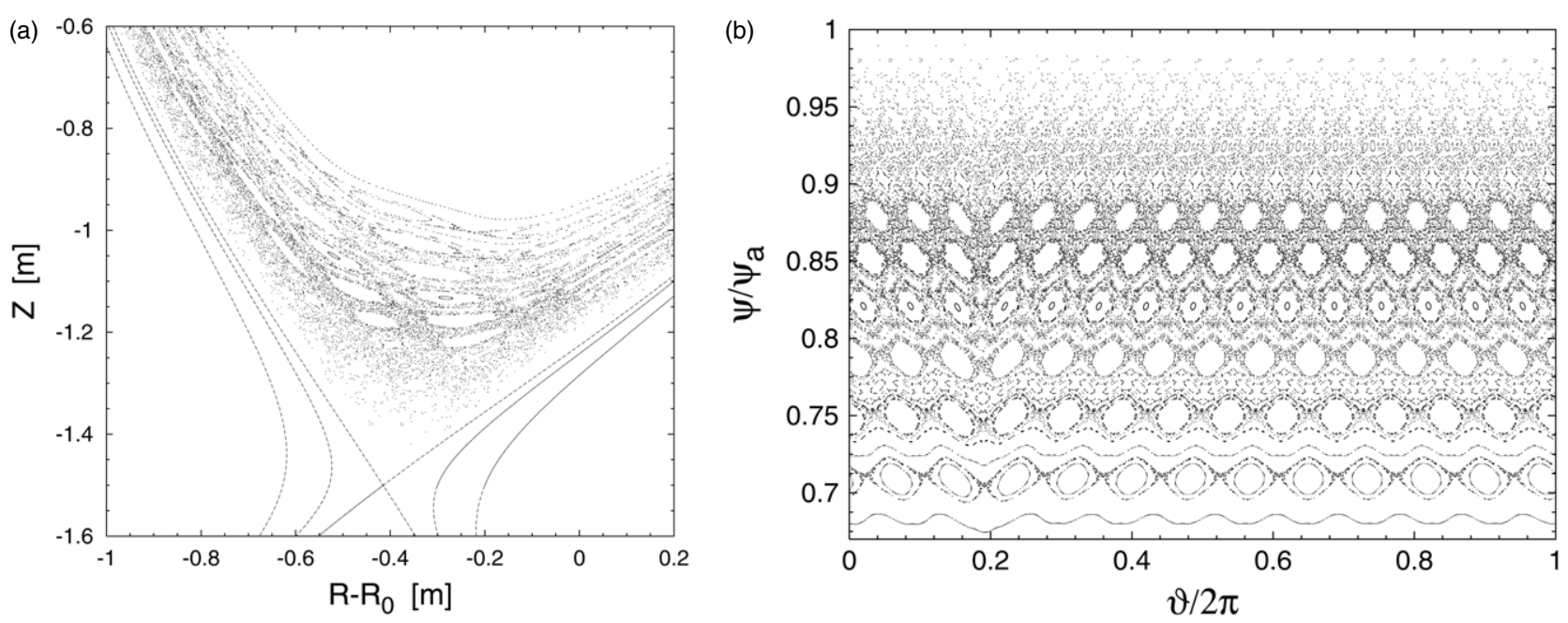

Figure 13. Poincare section of field lines in the poloidal plane $\varphi=0$ : $(a)$ near the X-point and $(b)$ in the $\left(\vartheta, \psi / \psi_{a}\right)$ plane. Perturbation current $I_{c}=100 \mathrm{kA}\left(\epsilon=I_{c} / I_{p}=0.05\right)$.

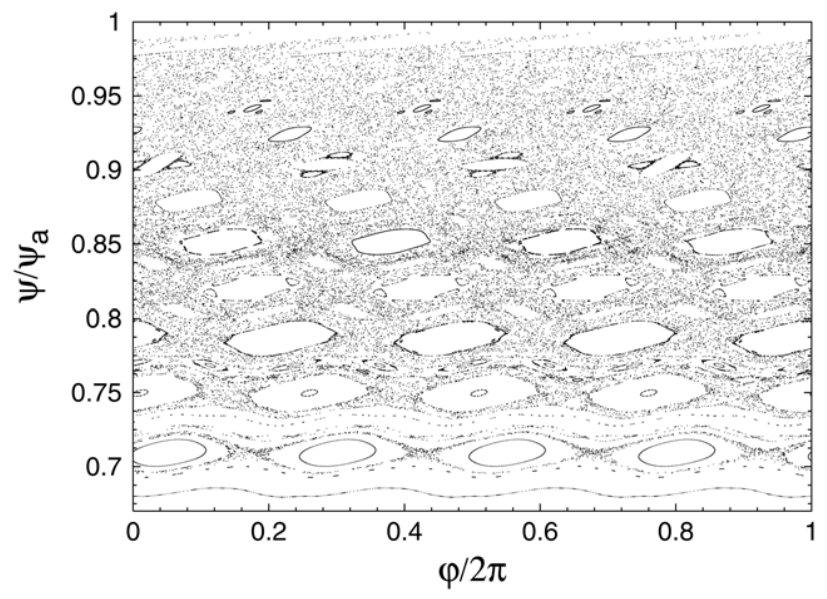

Figure 14. The same as in figure 13 but in the plane $\left(\varphi, X=R_{0} \xi\right.$ $(\xi>0)$. In the plot the coordinate $X$ is replaced by the normalized toroidal flux $\psi / \psi_{a}$.

Poincaré sections obtained in both the cases coincide except at the very edge of the plasma near the separatrix where field lines leave the plasma region. Field lines are terminated when they leave the plasma region.

In figure 13(a) shows the Poincaré section of field lines near the X-point in the real coordinate plane $(R, Z, \varphi=0)$, and $(b)$ shows a similar plot in the intrinsic coordinate plane $\left(\vartheta, \psi / \psi_{a}, \varphi=0\right)$. The Poincaré section in the $\left(\varphi, X=R_{0} \xi\right)$ plane is shown in figure 14 where the normalized toroidal flux, $\psi / \psi_{a}$, is used instead of the coordinate $X$.

As seen from figures $13(b)$ and 14 for the perturbation current $I_{c}=100 \mathrm{kA}$ the stochastic layer near the separatrix covers the sufficiently large area $\psi_{b}<\psi<\psi_{a}$ where the lower boundary $\psi_{b}$ is approximately equal to $0.73 \psi_{a}$, i.e. the stochastic layer is about $27 \%$ of the total toroidal flux. The details of the stochastic layer can be seen more clearly from the Poincaré section in the $(\varphi, \psi)$ plane. It is not uniformly chaotic: there are islands of stable motion, the socalled KAM (Kolmogorov-Arnold-Moser)-stability islands, embedded into the chaotic sea. They are remnants of islands on the resonant magnetic surfaces. Open chaotic field lines connecting the divertor plates may have very long connection lengths due to the trapping in these islands. The sizes of these islands decrease when approaching the separatrix. At the area very close to the separatrix there are four stripes along with field lines leaving the plasma region. This structure is clearly seen in figure 14. The direction of stripes in the Poincare sections is determined by the iteration direction of the mapping. Figure 14 describes when field lines are iterated in the positive direction of $\varphi$. The direction of stripes changes to the opposite if field lines are run in the negative direction of $\varphi$.

The fine structure of the stochastic layer in the area close to the separatrix and stripes can be revealed by plotting the so-called laminar plot. It is known that chaotic field lines in poloidal divertor tokamaks similar to the ones in ergodic divertor tokamaks can be considered as a chaotic scattering system $[18,19,31]$ (see [35-37] on chaotic scattering systems). In such systems an orbit may enter the system and leave it in one of several ways. The space of initial conditions corresponding to the different exit ways is separated by a boundary which may be fractal (see [38]).

Open field lines in poloidal tokamaks can be classified by a number of poloidal turns, $N_{p}$, necessary to connect the left and right divertor plates (see figure 12). The set of initial conditions for which field lines have a particular value $N_{p}$ is referred to as the basin. The fine structure of the stochastic layer can be seen on contour plots of basin boundaries with numbers $N_{p}$. These plots are referred to as laminar plots. The separatrix mapping (38) is a most computationally effective tool for calculating $N_{p}$. One step of the separatrix map corresponds to the one in the poloidal turn. It runs almost three orders faster than the iterative mapping. The laminar plot is obtained using the same procedure as in [31]: a field line with a particular initial coordinate $\left(\varphi_{i}, h_{i}\right)$ on the $(\varphi, h)$-plane is traced by the forward and backward separatrix maps (38) along the positive and the negative directions of the toroidal angle $\varphi$, respectively, until it reaches the divertor plates. The complete number of map iterations gives the number of poloidal turns, $N_{p}$, since a field line makes a full poloidal turn after each map iteration. 

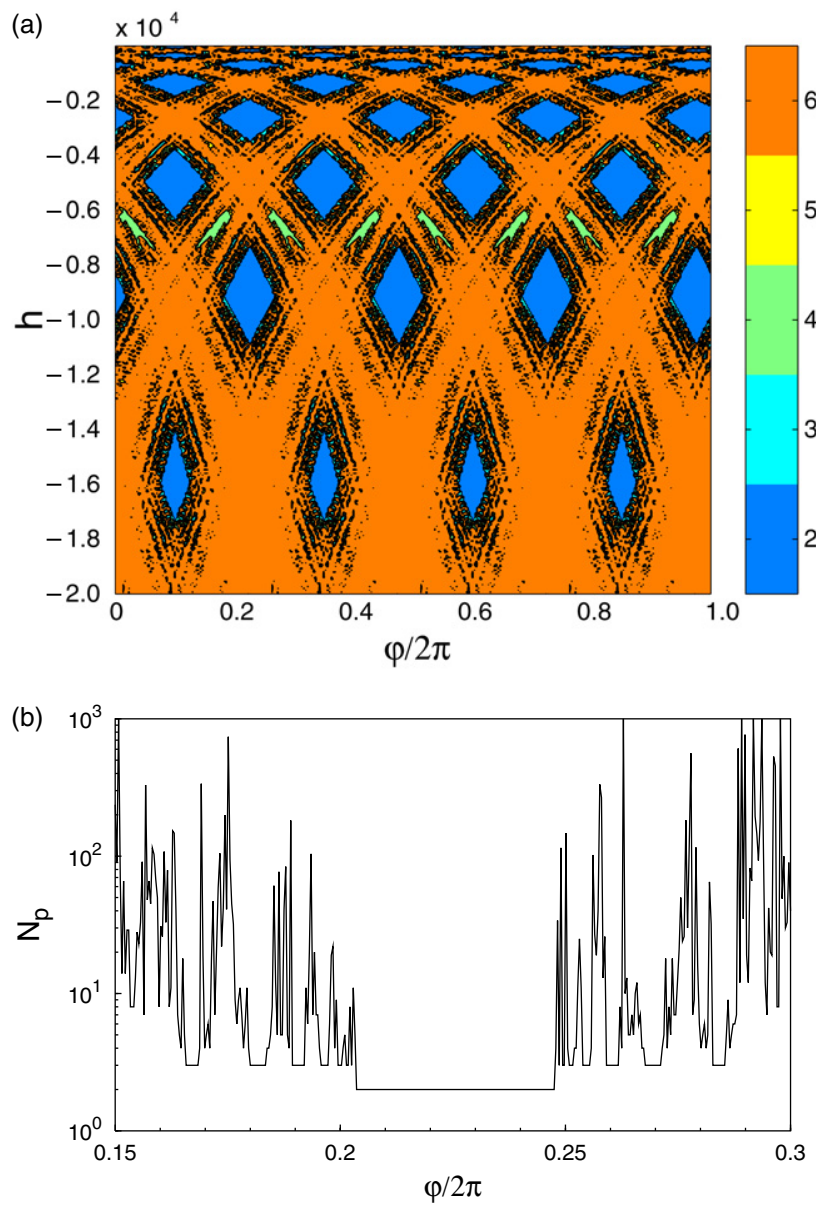

Figure 15. (a) Basin boundary structure of $N_{p}$ on the $(\varphi, h)$ plane $(h<0)$ of section $\Sigma_{s}$. (b) Toroidal dependence of $N_{p}$ in the region $54^{\circ}<\varphi<108^{\circ}$ along line $h=-1 \times 10^{-4}$ which corresponds to $X=0.0595 \mathrm{~m}$. The bottom value of $h=-2 \times 10^{-4}$ corresponds to $\psi=0.9872 \psi_{a}$.

The basin boundary structure of $N_{p}$ in the $(\varphi, h)$-plane lying on the upper branch of section $\Sigma_{s}$ is shown in figure 15(a). The values of the parameters chosen are the same as in the Poincaré section of field lines in figure 14. The lowest colour bar corresponds to the basins with the smallest number of $N_{p}=2$, and the highest one corresponds to the basins with $N_{p} \geqslant 8$. The latter basins are not resolved. Fine details of these basin boundaries are seen in figure $15(b)$ where the toroidal dependence of $N_{p}$ along the straight line $h=-10^{-4} \mathrm{~m}$ is shown. This line corresponds to $X=0.0595 \mathrm{~m}$. (The value $h=-2 \times 10^{-4}$ corresponds to $\psi=0.9872 \psi_{a}$.)

The structure of basin boundaries is similar to the corresponding structure in ergodic divertor tokamaks (see [31]. The regions with small connection numbers, $N_{p}=2,3,4$ cover relatively large areas. The boundaries of basins with higher numbers $N_{p}$ have a layered fractal structure.

\subsection{Structure of magnetic footprints}

The pattern of the crossing points of field lines with the divertor plates is known as magnetic footprints. The latter determine heat and particle deposition patterns on the divertor plates since electrons and ions predominantly follow the magnetic

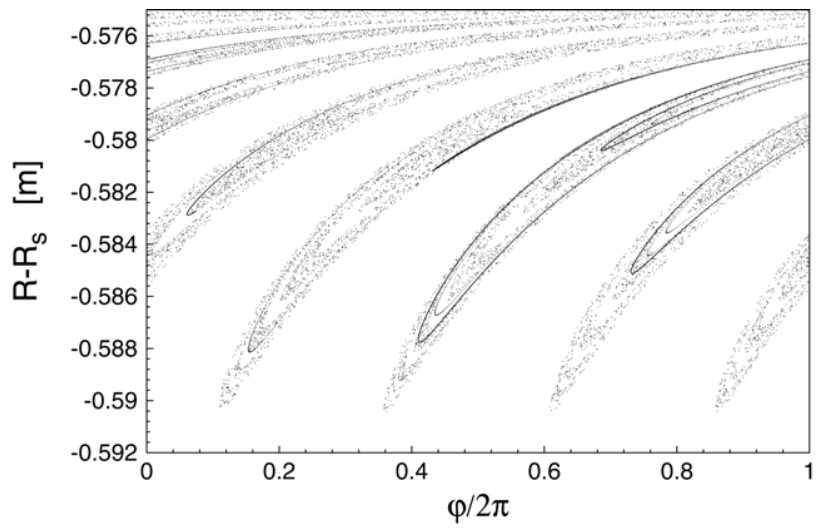

Figure 16. Magnetic footprints on the left divertor plate. The plasma and perturbation parameters are the same as in figure 13 and $X_{d}=0.2 \mathrm{~m}$.

field lines. This fact has been confirmed in many experimental observations performed in the DED-TEXTOR $[39,40]$.

In order to obtain the magnetic footprints on the divertor plates of poloidal tokamaks we used the following procedure. For simplicity we have supposed the simple geometry of divertor plates shown in figure 12. They consist of two segments parallel to the $\eta$-axis and located at distance $X_{d}$. The two branches of the unperturbed separatrix cross the divertor plates at $R=R_{s}$. We have taken a set of field lines with initial coordinates $\left(\varphi_{0}, h_{0}\right),\left(h_{0}<0\right)$, on the $(\varphi, Z)$-plane located in the stochastic layer of the plasma region. In order to obtain magnetic footprints on the left divertor plate field lines were traced using the forward separatrix map (38) until $h_{k}>0$, i.e. the field lines intersect the left branch of the section $\Sigma_{s}$. Then the field line with $\left(\varphi_{k}, h_{k}\right)$ is mapped to the divertor plane using mapping (39). In the latter mapping the increment of the toroidal angle, $\Delta \varphi$, for the geometry of the divertor plates shown in figure 12 is determined by (15), where $\xi=X_{d} / R_{0}$. The radial coordinate $R$ of the footprint can be found using the relation (13) between the relative poloidal flux, $h_{d}$, and the unperturbed Hamiltonian, i.e. $f\left(R_{d}\right) \equiv H_{0}\left(z_{d}, p_{z}\left(R_{d}\right)\right)-$ $H_{0}\left(z_{s}, p_{s}\right)=h_{d}$, where $p_{z}(R)=\ln \left(R / R_{0}\right) \approx\left(R-R_{0}\right) / R_{0}$. If the divertor plates are close to the $\mathrm{X}$-point then according to (11) we have $R_{d}=R_{0}-\sqrt{2 h_{d} R_{0}^{2} / \gamma+X_{d}^{2}}$. A set of crossing points, $\left(\varphi_{d}, h_{d}\right)$, on the divertor plate gives the magnetic footprint.

The magnetic footprint on the left divertor plate corresponding to the stochastic layer in figures 13 and 14 is shown in figure 16. The distance $X_{d}$ is taken equal to $0.2 \mathrm{~m}$. It consists of four clusters corresponding to the toroidal mode number $n=4$. A fine structure of the cluster shown in figure 17 represents the structure of boundaries of basins corresponding to the field lines with the different poloidal numbers $N_{p}$ : (a) shows the contour plot of $N_{p}$ in the $(\varphi, R)$ plane; $(b)$ the same but the $(X, Y)=\left(\left(R-R_{S}\right) \cos \varphi,\left(R-R_{S}\right) \sin \varphi\right)$ plane and (c) describes a toroidal dependence of $N_{p}$ along the straight line $R=R_{0}-0.583 \mathrm{~m}$ on the left divertor plate. As seen from figure 17 each cluster has a generic spiral-like structure with spirals corresponding to the different $N_{p}$.

The white area on the divertor plate in figure 16 and the area with $N_{p}=1$ in figure 17 correspond to field lines connecting the divertor plates without entering the plasma 

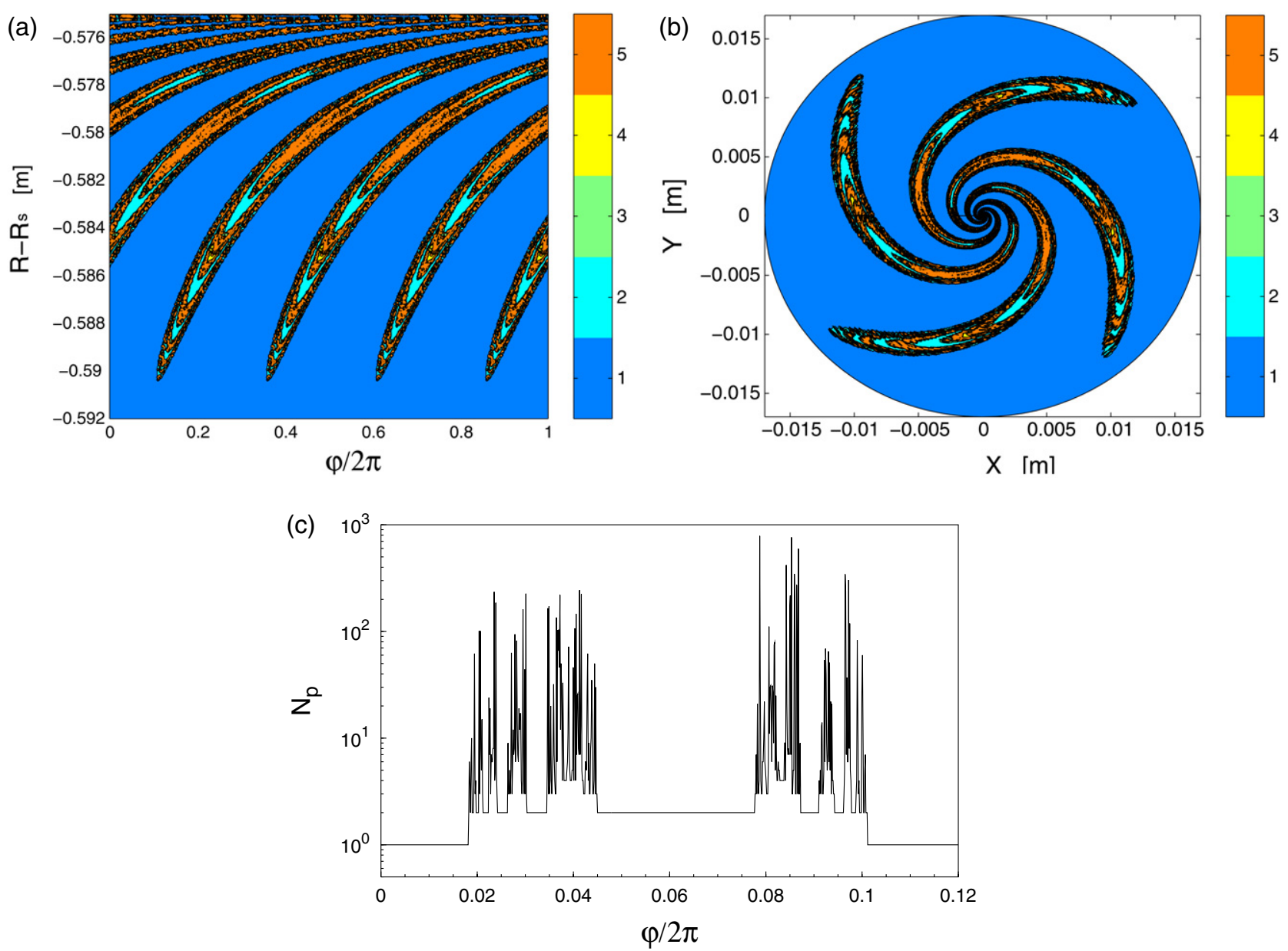

Figure 17. Basin boundary structure of $N_{p}$ on the divertor plate: $(a)$ in the $(\varphi, R)$ plane; $(b)$ in the $(X, Y)=\left(\left(R-R_{s}\right) \cos \varphi\right.$,

$\left.\left(R-R_{s}\right) \sin \varphi\right)$ plane and $(c)$ expanded view of the structure along the straight line $R=R_{0}-0.583 \mathrm{~m}$ covering the interval, $\left[0^{\circ}, 43.2^{\circ}\right]$, of the toroidal angle $\varphi$.

region. The areas with $N_{p} \geqslant 2$ correspond to field lines entering the plasma region. The radius of spirals presented in figures 16 and $17(a)$ and $(b)$ is about $1.2 \mathrm{~cm}$, while the gyroradii of thermal electrons and ions are $\rho_{e} \approx 10^{-3} \mathrm{~cm}$ and $\rho_{i} \approx 10^{-1} \mathrm{~cm}$ (for electron and ion temperatures $T_{e}=$ $T_{i}=100 \mathrm{eV}$ and the toroidal field $B_{t}=2 \mathrm{~T}$ ). Therefore, the heat deposition pattern will be close to magnetic footprints. However, the cross-field diffusion of particles sweeps out the fine structure of magnetic footprints and broadens the spirals.

\subsection{Diffusion of field lines in the stochastic layer}

In general the global transport of field lines in the stochastic layer formed near the separatrix cannot be described as a pure diffusive process (see, e.g. [41]). It is because the stochastic layer is not fully chaotic, and it consists of the structured KAM stability islands where field lines can be trapped. Nevertheless in the regions of the stochastic layer with highly developed chaos one can locally describe the behaviour of field lines as a diffusion process. The corresponding local diffusion coefficients of field lines can be qualitatively determined in the quasilinear approximation. In this approximation the diffusion coefficient of the Hamiltonian system (19)-(21) is given by [23]

$$
D_{\psi}(h)=\frac{\left\langle(\Delta \psi)^{2}\right\rangle}{2 \Delta \varphi}=\pi \epsilon^{2} \sum_{m}\left|m H_{m n}(\psi)\right|^{2} \delta\left(\frac{m}{q(\psi)}-n\right) .
$$

According to the relation (47) $D_{\psi}(h)$ can be expressed through the single function $R_{n}(h)$ :

$$
D_{\psi}(h)=(4 \pi)^{-1} \epsilon^{2} n^{2} q(h)\left|R_{n}^{(\mathrm{reg})}(h)\right|^{2} .
$$

Using the relations (7), (10) between the variables $h$, $\psi$ and the coordinate $\xi=X / R_{0}$, one obtains $\Delta X=$ $R_{0} /\left[\sqrt{2\left|\lambda_{1} h\right|} q(h)\right], \Delta \varphi \approx \Delta l / R_{0}$, where $\Delta l$ is the length element of a field line. Then the diffusion coefficient of field lines in the geometrical coordinates takes the form

$$
\begin{aligned}
D_{\mathrm{FL}}(h) & =\frac{\left\langle(\Delta X)^{2}\right\rangle}{2 \Delta l}=\frac{R_{0}}{2\left|\lambda_{1} h\right| q^{2}} D_{\psi} \\
& =\frac{R_{0} \epsilon^{2} n^{2}}{8 \pi\left|\lambda_{1} h\right| q(h)}\left|R_{n}^{(\mathrm{reg})}(h)\right|^{2} .
\end{aligned}
$$

The dependence of the local diffusion coefficient, $D_{\mathrm{FL}}(h)$, on the noramalized toroidal flux $\psi / \psi_{a}$ is plotted in figure 18 . The corresponding Poincaré section of the stochastic layer was shown in figure 13 . For instance, the local diffusion coefficient $D_{\mathrm{FL}}=2.25 \times 10^{-6} \mathrm{~m}^{2} \mathrm{~m}^{-1}$ at $\psi=0.95 \psi_{a}$. 


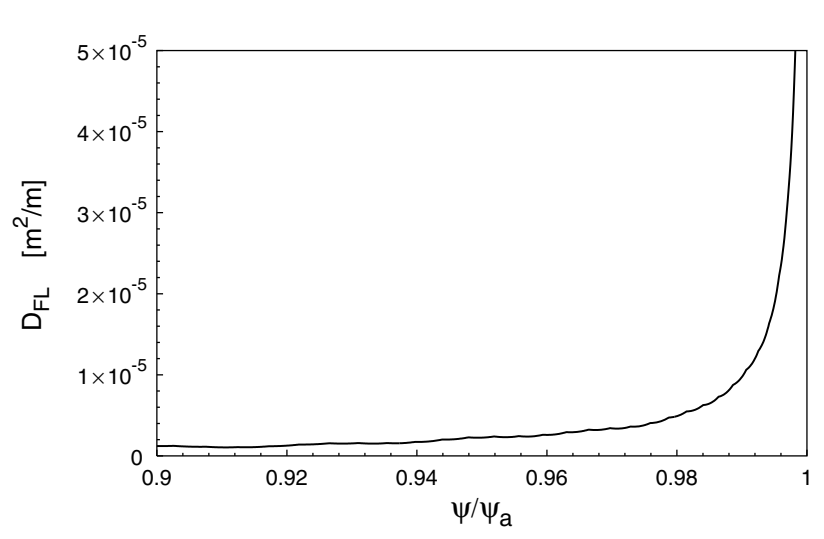

Figure 18. Local diffusion coefficients $D_{\mathrm{FL}}$ of field lines near the separatrix. Parameters are the same as in figure 13.

\section{Summary}

In summary, we described two mapping methods to study the stochastic field lines in poloidal divertor tokamak plasmas affected by external non-axisymmetic magnetic perturbations. The first mapping method is an iterative mapping of field lines along the toroidal angle. It is based on the canonical transformations of variables in the Hamiltonian equations of field lines in the Boozer coordinates. The mapping has a variable step along the toroidal angle, and its accuracy can be controlled by changing the mapping step.

The second method, the separatrix mapping, which has been applied to this problem is based on mapping of field lines in a certain section near the X-point after one full poloidal turn. It is a very effective tool to describe chaotic magnetic field lines near the separatrix and to obtain straightforwardly the magnetic footprints on the divertor plates. The separatrix map for arbitrary magnetic configuration has a generic form determined by only a few coefficients. These coefficients can be found from the equations of field lines, since one iteration of the separatrix map is equal to one (or half) poloidal turn it is almost by two-three orders faster than the direct numerical integration of the equations of field lines.

In this work we investigated only the structure of the magnetic field near the separatrix and on the divertor plates of tokamaks in the presence of external magnetic perturbations. The study of particle (electron and ion) motion in such perturbation fields in real tokamaks would require taking into account an electric field at the plasma edge. However, in order to tackle this important problem one should consider a drift motion of particles which is out of the scope of this paper.

\section{Acknowledgments}

This work has been partially supported by the project SFB 591 of the Deutsche Forschungsgemeinschaft (DFG).

\section{Reference}

[1] Wesson J. 2004 Tokamaks (Oxford Engineering Science Series, vol 48) 3rd edn (Oxford: Clarendon Press)
[2] Pomphrey N. and Reiman A. 1992 Phys. Fluids B 4 938-48

[3] Boozer A.H. and Rechester A.B. 1978 Phys. Fluids 21 682-9

[4] Reiman A. 1996 Phys. Plasmas 3 906-13

[5] Evans T.E., Moyer R.A. and Monat P. 2002 Phys. Plasmas 9 4957-67

[6] Evans T.E. et al 2004 Phys. Rev. Lett. 92235003

[7] Tomita Y., Seki S. and Momota H. 1977 J. Phys. Soc. Japan 42 687-93

[8] Tomita Y., Momota H. and Itatani R. 1978 J. Phys. Soc. Japan 44 637-42

[9] LaHaye R.J. 1991 Nucl. Fusion 31 1550-5

[10] Punjabi A., Verma A. and Boozer A.H. 1992 Phys. Rev. Lett. $693322-5$

[11] Punjabi A., Verma A. and Boozer A.H. 1994 J. Plasma Phys. 52 91-111

[12] Punjabi A., Verma A. and Boozer A.H. 1996 J. Plasma Phys. 56 569-603

[13] Punjabi A., Ali H. and Boozer A.H. 1997 Phys. Plasmas 4 337-45

[14] Punjabi A., Ali H. and Boozer A.H. 2003 Phys. Plasmas 10 3992

[15] Ali H., Punjabi A., Boozer A.H. and Evans T. 2004 Phys. Plasmas 111908

[16] Yamagishi T. 1995 Fusion Technol. 27 505-8

[17] Abdullaev S.S. and Zaslavsky G.M. 1995 Phys. Plasmas 2 4533-40

[18] Abdullaev S.S. and Zaslavsky G.M. 1996 Phys. Plasmas 3 516-28

[19] Abdullaev S.S. and Finken K.H. 1998 Nucl. Fusion 38 531-44

[20] Chirikov B.V. 1979 Phys. Rep. 52265

[21] Abdullaev S.S. 2004 Phys. Rev. E 70046202

[22] Abdullaev S.S. 2005 Phys. Rev. E 72046202

[23] Lichtenberg A.J. and Lieberman M.A. 1992 Regular and Stochastic Motion 2nd edn (New York: Springer)

[24] Melnikov V.K. 1963 Trans. Moscow Math. Soc. 12 1-56

[25] Abdullaev S.S. 1999 J. Phys. A: Math. Gen. 322745

[26] Abdullaev S.S. 2002 J. Phys. A: Math. Gen. 352811

[27] Abdullaev S.S. 2004 Nucl. Fusion 44 S12

[28] Finken K.H. (ed) 1997 The dynamic ergodic divertor Fusion Eng. Des. 37 335-448 (Special issue)

[29] Abdullaev S.S., Finken K.H. and Spatschek K.H. 1999 Phys. Plasmas 6 153-74

[30] Finken K.H., Abdullaev S.S., Kaleck A. and Wolf G.H. 1999 Nucl. Fusion 39637

[31] Abdullaev S.S., Eich Th. and Finken K.H. 2001 Phys. Plasmas 82739

[32] Abdullaev S.S., Finken K.H., Jakubowski M.W., Kasilov S.V., Kobayashi M., Reiser D., Reiter D., Runov A.M. and Wolf R. 2003 Nucl. Fusion 43299

[33] Morozov A.I. and Solov'ev L.S. 1966 Reviews of Plasma Physics vol 2, ed M.A. Leontovich (New York: Consultants Bureau) pp 1-101

[34] Zaslavsky G.M., Sagdeev R.Z., Usikov D.A. and Chernikov A.A. 1991 Weak Chaos and Quasiregular Patterns (Cambridge: Cambridge University Press)

[35] Eckhard B. 1988 Physica D 3389

[36] Bleher S., Ott E. and Grebogi C. 1989 Phys. Rev. Lett. 63919

[37] Tel T. and Ott E. 1993 Chaos 3417

[38] Bleher S., Grebogi C. and Ott E. 1990 Physica D 4687

[39] Jakubowski M., Abdullaev S.S. and Finken K.H. 2004 Nucl. Fusion $44 \mathrm{~S} 1-11$

[40] Abdullaev S.S. et al 2004 Proc. Joint Varenna-Lausanne Int. Workshop on Theory of Fusion Plasmas (Varenna, Italy, 30 August-3 September 2004) eds J.W. Connor et al (ISPP-21) (Bologna: SIF, Piero Caldirola) p 373

[41] Abdullaev S.S. 2000 Phys. Rev. E 623508 